\title{
X-ray variability of NGC 2516 stars in the XMM-Newton observations ${ }^{\star}$
}

\author{
A. Marino ${ }^{1}$, G. Micela ${ }^{2}$, I. Pillitteri ${ }^{1}$, and G. Peres ${ }^{1}$ \\ 1 DSFA, Università di Palermo, Piazza del Parlamento 1, 90134 Palermo, Italy \\ e-mail: marino@astropa.unipa.it \\ 2 INAF - Osservatorio Astronomico di Palermo, Piazza del Parlamento 1, 90134 Palermo, Italy \\ Received 9 December 2005 / Accepted 1 May 2006
}

\section{ABSTRACT}

\begin{abstract}
We present the characteristics of the X-ray variability of stars in the cluster NGC 2516 as derived from XMM-Newton/EPIC/pn data. The X-ray variations on short (hours), medium (months), and long (years) time scales have been explored. We detected 303 distinct X-ray sources by analysing six EPIC/pn observations; 194 of them are members of the cluster. Stars of all spectral types, from the early-types to the late-M dwarfs, were detected. The Kolmogorov-Smirnov test applied to the X-ray photon time series shows that, on short time scales, only a relatively small fraction (ranging from $6 \%$ to $31 \%$ for $\mathrm{dG}$ and $\mathrm{dF}$, respectively) of the members of NGC 2516 are variable with a confidence level $\geq 99 \%$; however, it is possible that the fraction is small only because of the poor statistics. The time X-ray amplitude distribution functions (XAD) of a set of dF7-dK2 stars, derived on short (hours) and medium (months) time scales, seem to suggest that medium-term variations, if present, have a much smaller amplitude than those on short time scales; a similar result is also obtained for $\mathrm{dK} 3-\mathrm{dM}$ stars. The amplitude variations of late-type stars in NGC 2516 are consistent with those of the coeval Pleiades stars. Comparing these data with those of ROSAT/PSPC, collected 7-8 years earlier, and of ROSAT/HRI, just 4-5 years earlier, we find no evidence of significant variability on the related time scales, suggesting that long-term variations due to activity cycles similar to the solar cycle are not common among young stars. Indications of spectral variability was found in one star whose spectra at three epochs were available.
\end{abstract}

Key words. X-ray: stars - stars: activity - stars: early-type - stars: late-type - galaxy: open clusters and associations: individual: NGC 2516 - stars: coronae

\section{Introduction}

Observations using the Einstein observatory, nearly 30 years ago, showed the ubiquity of stellar X-ray emission throughout the HR diagram (Vaiana et al. 1981). X-ray emission from stars has been attributed to several physical mechanisms depending on the mass of the star. Going from high to low mass, they include winds, dynamo, and turbulent dynamo. Variability studies are one of the best ways to explore these mechanisms and to infer physical conditions of the regions where X-ray emission originates and their evolution along stellar life (e.g. Montmerle et al. 1983; Ambruster et al. 1987; Stern et al. 1995; Marino et al. 2003a,b; Pillitteri et al. 2005). In this context, open clusters, providing large, chemically homogeneous, and precisely dated samples of stars, are ideal laboratories for studying coronal emitters and for constraining the X-ray-generating mechanisms. Comparative studies of the variability properties of homogeneous classes are excellent for diagnosing and determining the origin of the X-ray emission.

In this perspective we have analysed the X-ray variability properties of homogeneous samples (with respect to mass and age): dF7-dK2 and dK3-dM stars in the solar neighbourhood, dF7-dK2, and dK3-dM of Pleiades observed with ROSAT-PSPC (Marino et al. 2000, 2002, 2003b). These studies show that the short-term variability of solar-type stars decreases with age (together with luminosity), while long-term variability increases. In contrast, the short-term variability of low mass stars is present

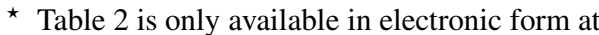
http://www.edpsciences.org in all stellar life phases while long-term variations have never been observed.

Also called the "Southern Pleiades", NGC 2516 is a rich and young open cluster in the constellation of Carina, that has been observed several times at the XMM-Newton observatory, thereby allowing us the study of X-ray variability properties on multiple time scales. At a distance of less than 400 pc (387 pc, Jeffries et al. 1997; 346 pc, Robichon et al. 1999), the cluster contains about 1300 known members spanning all spectral types and enabling simultaneous study of the different processes driving X-ray emission from stars with different internal structures. The metallicity of the cluster is controversial; several photometric studies have suggested a metal underabundance by a factor of 2 with respect to the Sun (e.g. Jeffries et al. 1997, 1998, 2001; Pinsonneault et al. 1998), while Terndrup et al. (2002) have found solar-like metal abundance on the basis of high-resolution spectra. Since metallicity affects the depth of the convection zone, which in turn should influence the dynamo's efficiency, observable differences in the X-ray emission levels may be expected between solar and sub-solar abundances (Micela et al. 2000). The ROSAT observations have shown that the $G$ and $K$ members in NGC 2516 were underactive in X-rays with respect to the Pleiades $\mathrm{G}$ and $\mathrm{K}$ stars, while no difference was found for M stars. As discussed by Jeffries et al. (1997) and Micela et al. (2000), these results could be attributed to a low-metallicity effect, although when taking the recent optical data into account (Terndrup et al. 2002) these explanations are questioned. Also a recent analysis of summed XMM/EPIC data (Pillitteri et al. 2006) shows that late-types stars in NGC 2516 are significantly less luminous than those of the Pleiades. 
Table 1. XMM-Newton/EPIC/pn observations of NGC 2516.

\begin{tabular}{cccccccc}
\hline \hline Obs. & Obs. Id & $\begin{array}{c}\text { Orbit } \\
\text { No. }\end{array}$ & $\begin{array}{c}\text { Filtered } \\
\text { time [ks] }\end{array}$ & $\begin{array}{c}\text { Start } \\
\text { date }\end{array}$ & $\begin{array}{c}\text { No. of X-ray } \\
\text { sources }\end{array}$ & $\begin{array}{c}\text { No. of detected } \\
\text { members }\end{array}$ & $\begin{array}{c}\text { No. of optical } \\
\text { members }\end{array}$ \\
\hline$a$ & 0113891001 & 060 & 19.8 & 2000 April 06 & 128 & 87 & 409 \\
$b$ & 0113891101 & 060 & 14.3 & 2000 April 07 & 118 & 73 & 408 \\
$c$ & 0126511201 & 092 & 22.3 & 2000 June 10 & 184 & 128 & 419 \\
$d$ & 0134531201 & 209 & 18.8 & 2001 January 29 & 174 & 121 & 408 \\
$e$ & 0134531301 & 209 & 6.0 & 2001 January 29 & 95 & 68 & 402 \\
$f$ & 0134531501 & 346 & 18.5 & 2001 October 29 & 168 & 112 & 423 \\
\hline
\end{tabular}

NGC 2516 has been observed several times with Chandra and the analysis of the observations was made by Harnden et al. (2001) and Damiani et al. (2003). Wolk et al. (2004) used Chandra data for a timing analysis of NGC 2516 stars, finding that the stochastic variability rate is similar for all sources in their sample, while the time scale of variability is shorter for later-type stars.

In this paper we present a study of the X-ray variability of the NGC 2516 stars analysing six XMM-Newton/EPIC observations spanning $\sim 19$ months. We explore X-ray variability properties on short (hours), medium (months), and long (years) time scales, we compare our data with those obtained for the coeval Pleiades, and we search for spectral variability.

The structure of the paper is the following: Sect. 2 describes the XMM-Newton/EPIC observation set and the data analysis; Sects. 3 and 4 present the time and the spectral analysis, respectively; Sect. 5 summarises the main results.

\section{X-ray observations and data analysis}

NGC 2516 was observed many times during the first two years of satellite calibration operations. We used only EPIC-pn data detector, since the MOS data have a lower statistic than pn. The characteristic of the six EPIC/pn observations are summarised in Table 1. The progressive letter in Col. 1 is a reference to Table 2. The first two observations were centred at 7:58:20, $-60: 52: 13(\mathrm{~J} 2000)$ and the remaining at 119.58:22, $-60: 45: 36$ (J2000). All six observations that we consider were performed with the thick filter. The data span 19 months with exposure time ranging from $6 \mathrm{ks}$ to $\sim 22 \mathrm{ks}$. All pn data were processed using the XMM-Newton Science Analysis System (SAS) 6.0.0. We used the epchain task to process the EPIC/pn observation data file, obtaining six lists with time, position, and energy of the events recorded in the pn detector. To minimise the background due to non-X-ray events, we retained only single, double, triple, and quadruple pixel events in the $0.3-5.5 \mathrm{keV}$ band. We limited the energy band to $0.3-5.5 \mathrm{keV}$, since data below $0.3 \mathrm{keV}$ are contaminated by low-energy electronic noise events, while background counts dominate above $5.5 \mathrm{keV}$ for coronal sources (e.g. Read \& Ponman 2003). Furthermore, we filtered the data to maximise the signal-to-noise ratio and to minimise the so-called proton flare phenomenon, which produces an enhancement of noise due to protons "focused" by XMM-Newton mirrors and essentially indistinguishable from bona-fide X-ray events. To this end we applied a technique developed at INAF - Osservatorio Astronomico of Palermo (Sciortino et al. 2002) that maximises the statistical significance of weak sources by identifying and removing fractions of the exposure time strongly affected by highbackground episodes.

\subsection{Source detection}

Source detection and X-ray photometry were obtained in the 0.3-5.5 keV bandpass using the wavelet detection code developed at the INAF - Osservatorio Astronomico di Palermo and based on the algorithm previously developed for the ROSAT/PSPC (Damiani et al. 1997a,b) and adapted to the XMM-Newton case. Large sets of simulations of pure background signal were performed to derive the appropriate detection threshold that limits the number of spurious detections. We adopted a threshold that statistically retains only one spurious source per field.

An exposure map for each observation was created with the SAS task eexpmap to perform this analysis. For each of the six observations, we have found a number of X-ray sources ranging from 95 (Obs. $e$ in Table 1) to 184 (Obs. $c$ in Table 1). In order to find the X-ray multiply observed sources, we crossmatched the detections of each observation with all the others adopting a threshold of $20^{\prime \prime}$, derived by taking the formal error of the detected source positions into account. However we found that more than $90 \%$ of the cross-matchs are within $8^{\prime \prime}$. We also found that 42 sources were detected six times, 23 five times, 39 four times, 40 three times, 60 two times, and 99 just one.

\subsection{Cluster members and identifications}

We compiled an optical catalog of cluster members based on the list of 1254 stars compiled by Jeffries et al. (2001) and based on $B, V$, and $I$ photometry. Furthermore we appended 43 cluster stars brighter than $V=9.8$ that were not present in the former sample. The number of X-ray sources identified as optical members are reported in Col. 7 of Table 1 for each of the six observations; the number of the optical members in each observation is reported in Col. 8. The cross-identification between X-ray and optical sources for each of the six observations was made in two steps: in the first we searched for a systematic offset between $\mathrm{X}$-ray source positions and the optical position of the members. In the second, we corrected the X-ray positions for this systematic offset, before matching the X-ray and optical member positions, and then retained an identification if the offset between X-ray and optical positions was less than $8^{\prime \prime}$. The choice of such a limiting distance is a good compromise between the attempt to minimise the number of spurious identifications for an offset bona-fide optical counterparts. For 8 cases it was impossible to resolve very close stars, owing to the limited spatial resolution of the X-ray telescope. For these unresolved sources, the X-ray flux was divided evenly between the optical candidates in the absence of more information.

Following Damiani et al. (2003), we attribute spectral types that use $B-V$ and $V-I$ optical colours corrected for the average cluster reddening $E(B-V)=0.12$. Detected members cover the whole mass range present in the optical catalog. Among these stars we considered in the following variability studies all those 
Table 3. Results of the K-S test for each X-ray detected source.

\begin{tabular}{cccc}
\hline \hline $\begin{array}{c}\text { Confidence }^{1} \\
\text { level }\end{array}$ & $\begin{array}{c}\text { Number of total } \\
\text { sources }\end{array}$ & $\begin{array}{c}\text { Number of cluster } \\
\text { members }\end{array}$ & Number of non members \\
\hline$<90 \%$ & $490(74 \%)$ & $345(73 \%)$ & $145(79 \%)$ \\
$90 \%-99 \%$ & $96(15 \%)$ & $72(15 \%)$ & $24(13 \%)$ \\
$\geq 99 \%$ & $71(11 \%)$ & $57(12 \%)$ & $14(8 \%)$ \\
\hline
\end{tabular}

${ }^{1}$ Confidence level for the rejection of the constant source hypothesis.

Table 4. Results of the K-S test for the stars of NGC 2516 grouped by spectral type.

\begin{tabular}{ccccrcc}
\hline \hline Confidence $^{1}$ & \multicolumn{5}{c}{ Number of cluster members } \\
level & $\mathrm{B}$ & $\mathrm{dA}$ & $\mathrm{dF}$ & $\mathrm{dG}$ & $\mathrm{dK}$ & $\mathrm{dM}$ \\
\hline$<90 \%$ & $9(64 \%)$ & $62(74 \%)$ & $9(69 \%)$ & $109(83 \%)$ & $119(70 \%)$ & $37(59 \%)$ \\
$90 \%-99 \%$ & $2(14 \%)$ & $13(15 \%)$ & 0 & $14(11 \%)$ & $32(19 \%)$ & $11(17 \%)$ \\
$\geq 99 \%$ & $3(22 \%)$ & $9(11 \%)$ & $4(31 \%)$ & $8(6 \%)$ & $18(11 \%)$ & $15(24 \%)$ \\
\hline
\end{tabular}

${ }^{1}$ Confidence level for the rejection of the constant source hypothesis.

sources with more than 25 total counts in a single pn observation, for a total of 474 detections. Table 2 summarise their optical and X-ray characteristics.

\section{Time variability}

The analysis of all observations of NGC 2516 allow us to explore the X-ray variability on time scales that range from hours to 19 months. We obtained light curves in the $0.3-5.5 \mathrm{keV}$ band for all detected X-ray sources by extracting the photon arrival time within circular regions selected interactively using Astronomical Data Visualization DS9 display software and subtracted from an area-scaled background. We adopted a radius of 3.5 times the radius determined by the PWDetect algorithm to select sources and background regions. In some cases we used a smaller radius to exclude contributions from nearby stars. In general, detections have relatively low statistics with $90 \%$ of the detections having less than 190 counts and only 23 detections more than 300 counts.

In order to have a statistical evaluation of the X-ray variability, we applied the unbinned Kolmogorov-Smirnov (K-S) test to the X-ray photon time series of our detected sources for each observation. Column 10 of Table 2 reports the results in terms of the confidence level at which we can reject the hypothesis that the source in the given observation is constant. Since the exposure times of the observations are in the 6-22.3 ks range, we will refer to this analysis as short-time- scale variability. For each observation, we also ran the K-S test on the counts detected in the background regions to monitor possible background variability. In the few cases in which the background counts are variable with a confidence level (CL) $>99 \%$, the background counts are much lower than those of the source, making us confident of the results of the test variability.

Table 3 summarises the K-S test results for the total sample of X-ray sources (Col. 2), for the members (Col. 3), and for nonmembers (Col. 4). Among cluster members, only a small fraction $(12 \%)$ of stars are variable with a $\mathrm{CL} \geq 99 \%$, while $73 \%$ are not variable $(\mathrm{CL}<90 \%)$; variability on a short-time-scale is not very common in NGC 2516, at least given the statistics of our observations. Table 4 and Fig. 1 summarise the K-S test results for member stars of different spectral types; $\mathrm{dF}$ stars are the sources with the highest rate of variability (although the sample is very small), $\mathrm{dG}$ are those that are less variable. In contrast, Wolk et al. (2004) find that the $\mathrm{dF}$ and $\mathrm{B}$ in their sample have

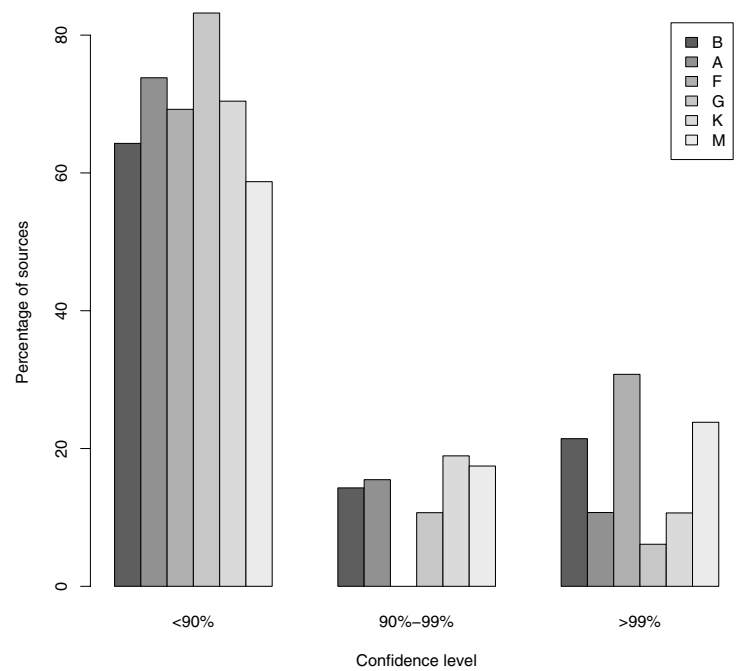

Fig. 1. Percentage of detected stars vs. variability confidence level and spectral type.

the lowest rate of variability. Frequency of the variability on a short-time-scale is approximately equal among late- and earlytypes stars. The fraction of sources with significant variability is very small for each spectral type suggesting that, on short time scales, the properties of variability do not depend strongly on the mass. However, the sensitivity to variability depends on count statistics, and the absence of variability in faint stars may be not intrinsic but instead due to low counting statistics. Also the comparisons of our results with those obtained for other sample stars are limited by different statistics.

\subsection{Time $X$-ray distribution functions}

We derived the time amplitude $\mathrm{X}$-ray luminosity distribution function (Time XAD) for the dF7-dK2 (0.5 $\leq B-V \leq$ $0.99)$ and dK3-dM $(B-V>0.9)$ stars as in Marino et al. (2003b). Time XAD yields the fraction of time that a star spends with a count rate higher, by a given factor, than its minimum value. This distribution is constructed by considering for each star the ratio between the count rate observed during a given observation and the minimum rate for that source. We computed the Time XAD on a given time scale considering only 


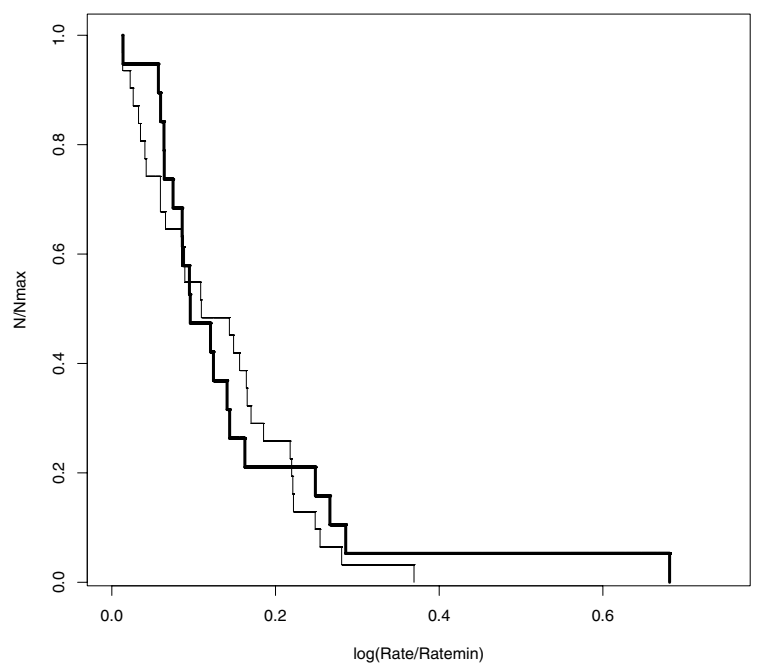

Fig. 2. The Time XAD for $\mathrm{dF} 7-\mathrm{dK} 2$ stars on short ( $\leq 1$ day, Obs. $a-b$, $d-e$, thin line) and long ( $\sim 17$ months, Obs. $c-f$, thick line) time scales.

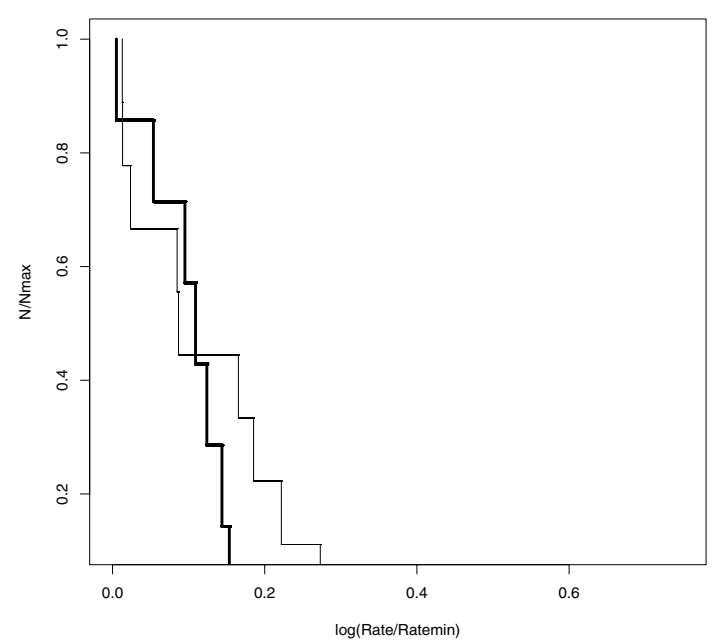

Fig. 3. As in Fig. 2 for dK3-dM on short (thin line) and long (thick line) time scales.

observations obtained at a time separation of the order of the time scale we want to study. For example, if we want to study a 1-day time scale, we can consider Obs. $a, b, d$, and $e$ of Table 1.

In Fig. 2 the Time XAD on short ( $<1$ day) time scale with that on a medium (17 months) time scale. The same plot for dK3-dM stars is shown in Fig. 3. To explore the presence of variations on medium time scale amplitudes, we excluded the stars variable $(\mathrm{CL} \geq 99 \%)$ on short time scales that could produce spurious variability. Both the dF7-dK2 and dK3-dM distributions on short and medium time scales appear very alike, indicating that medium-term variations, if they exist at all, must have a much larger amplitude than those on short time scales. The null hypothesis that the XADs on short and medium time scales of dF7-dK2 stars are drawn from the same parent distribution cannot be rejected.

\subsection{Long-term variability}

The Sun's 11-year activity cycle, as observed in optical, radio, ultraviolet, and X-ray bands, is a well-established phenomenon. In the soft X-ray bandpass, the flux varies by about one order of magnitude during the solar cycle (Kreplin et al. 1977; Peres et al. 2000). The amplitude variations of the Sun are strongly

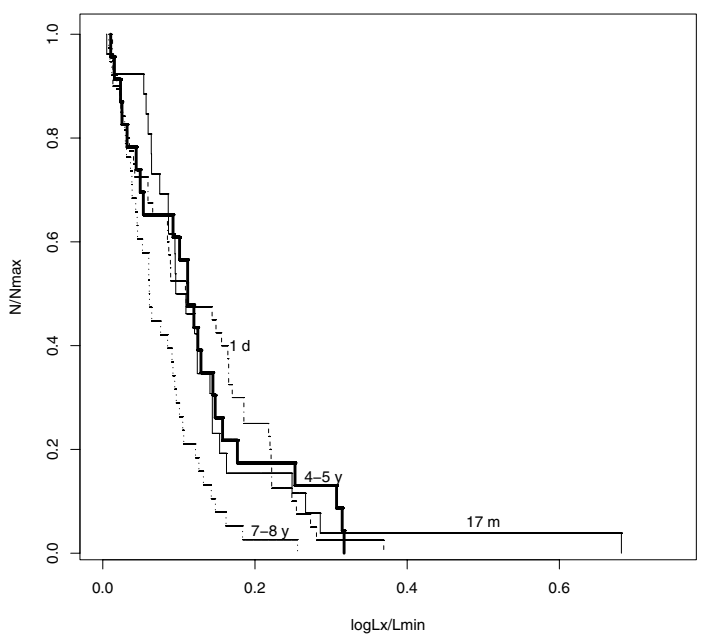

Fig. 4. The Time XADs for dF7-dM on 7-8 years (dotted line), on 4-5 years (thick line), on $\sim 17$ months (thin solid line) and 1 day (dotdashed line) time scales.

dependent on time scale, with the highest probability of observing high-amplitude variations, between 4 and 7.5 years (Micela \& Marino 2003). On these time scales, typically the observed variations are on average of the order of $1 \mathrm{dex}$, but the spread is very wide with an $80 \%$ probability of detecting variations in the $0.1-1.8$ dex range. Solar-like cycles have been observed in late-type stars from chromospheric flux variations (Wilson 1978; Baliunas et al. 1995). Only in more recent years, X-ray longterm variability possibly due to cyclical variations has been observed in stars (Hempelmann et al. 2003; Favata et al. 2004). By combining X-ray data obtained with ROSAT and, at present, XMM-Newton, we explored the long-term variability of the late-type stars of NGC 2516.

Thirty-eight late-type stars $(\mathrm{dF} 7-\mathrm{dM})$ of the cluster observed with XMM-Newton were observed with ROSAT/PSPC in 1993 (Jeffries et al. 1997) and twenty-three with ROSAT/HRI in 1997 (Micela et al. 2000), allowing us to explore variability on 7-8 and 4-5 year time scales, respectively. In order to compare our results with ROSAT observations, we derived EPIC/pn X-ray luminosities in the (0.15-2.0) keV bandpass, assuming a constant conversion factor to convert count rates to flux, as more extensively described in Sect. 3.3. For each star, several points were available from EPIC/pn and only one from PSPC ( $\left.L_{\mathrm{PSPC}}\right)$ and HRI $\left(L_{\mathrm{HRI}}\right)$. We reduced the influence of short and medium variability in the EPIC/pn data, averaging the latter to a single mean value $\left(L_{\mathrm{pn}}\right)$. The amplitude of variability is defined as $\log \left(L_{x} / L_{x \min }\right)$, where $L_{x}$ is the higher and $L_{x \min }$, the lower value between $L_{\mathrm{pn}}$ and $L_{\mathrm{PSPC}}$ and between $L_{\mathrm{pn}}$ and $L_{\mathrm{HRI}}$, respectively. Figure 4 shows the XADs of the stars observed both with PSPC and EPIC/pn observations, and with HRI and EPIC/pn. For comparison in the same figure, we also show the XADs limited to EPIC/pn dF7-dM stars on time scales of 1 day and on 17 months. The XADs on time scales of 4-5 years and on 7-8 years are marginally ( $C L \geq 96 \%)$ different, suggesting that long-term variations could be present. However, long-term variations, if they exist, must have a smaller amplitude than the short and medium term variations, or comparable to them. Our finding that there is no evidence of significant long-term variability agrees with those for other samples of young stars (e.g. Gagné et al. 1995; Marino et al. 2003b; Pillitteri et al. 2004; Stern et al. 1995; Marino et al. 2005), supporting a scenario in which stars much younger than the Sun (i.e. at ages $\lesssim 1 \mathrm{Gyr}$ ) do not have long-term 


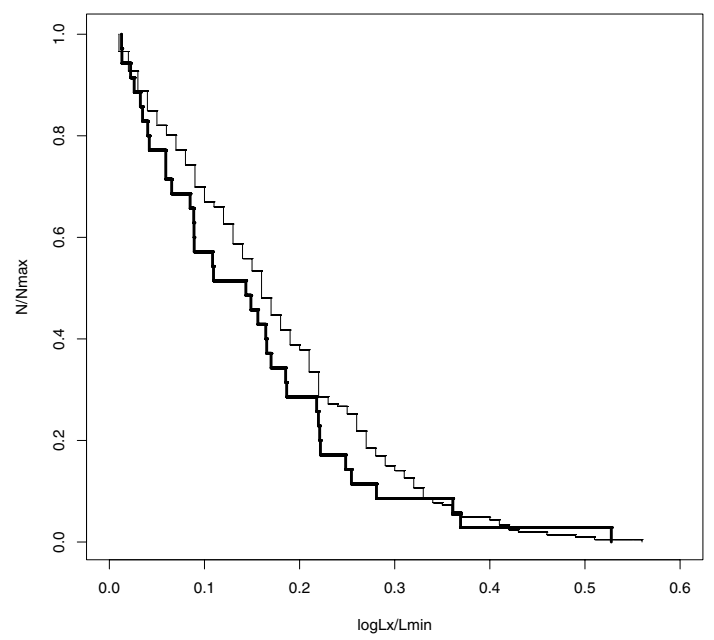

Fig. 5. The Time XAD for NGC 2516 (thick line) and Pleiades (thin line) dF7-dK2 stars.

cycles or their cycle amplitudes are much smaller than the solar one. On the other hand, there is growing evidence that cycles are present in older stars having age comparable to the solar one (Hempelmann et al. 2003; Favata et al. 2004).

\subsection{Comparison with the Pleiades}

The X-ray emission level decreases with increasing age as an effect of rotational braking (e.g. Micela et al. 1985, 1990, 1996; Barbera et al. 1993; Stauffer et al. 1994; Maggio et al. 1987). A decrease by three dex in the median value of $L_{x}$ with a spread of about 1 dex was observed in coeval stars. How much of this spread is due to variability and both if and how variability properties change with age is not clear. Comparing clusters of a very similar age is very useful for testing the evolutionary scenario. However ROSAT observations have shown that clusters of the same age may have significantly different levels of X-ray emission. For instance, late type-stars in the Praesepe cluster are much weaker emitter in X-rays than the Hyades stars (Randich et al. 1995), despite the fact that they have the same age and chemical composition as the Hyades. Many attempts have been made to explain the observed difference, but this puzzle has not yet been totally solved. An observation of the Praesepe cluster obtained with XMM-Newton (Franciosini et al. 2003) supports the hypothesis that Praesepe may be formed by two merging clusters with different ages.

We compared NGC 2516 with the slightly younger Pleiades; in particular, we compared the Time XADs of dF7-dK2 and dK3-dM members of NGC 2516 with the analogous ones for the Pleiades (Marino et al. 2003b). We converted EPIC/pn count rates to flux in the $0.1-2.4 \mathrm{keV}$ ROSAT energy band. In deriving $\mathrm{X}$-ray flux we assumed a constant conversion factor of $4.0 \times 10^{-12} \mathrm{erg} \mathrm{cm}^{-2} /$ count for the pn camera, computed with PIMMS, for a 1-T Raymond-Smith spectrum with a temperature of $\log T=6.80 \mathrm{~K}$, and a fixed $N_{\mathrm{H}}=7.5 \times 10^{20} \mathrm{~cm}^{-2}$ corresponding to the cluster extinction $A_{\mathrm{V}}=0.37$ (Jeffries et al. 2001). Figure 5 shows the Time XADs of dF7-dK2 in NGC 2516 and Pleiades: the two distributions appear marginally different, with the Pleiades dF7-dK2 amplitude of variations larger than those of NGC $2516 \mathrm{dF} 7-\mathrm{dK} 2$. However, using the two sample K-S test, the null hypothesis that the two distributions came from the same parent distribution, can be rejected only at a CL $\geq 73 \%$. Analogously, Time XADs of dK3-dM in NGC 2516 and

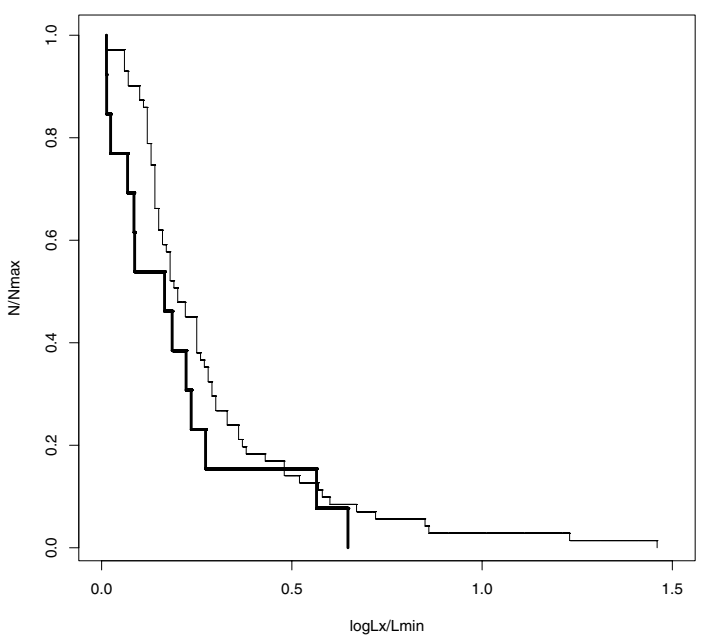

Fig. 6. As in Fig. 5 for dK3-dM stars.

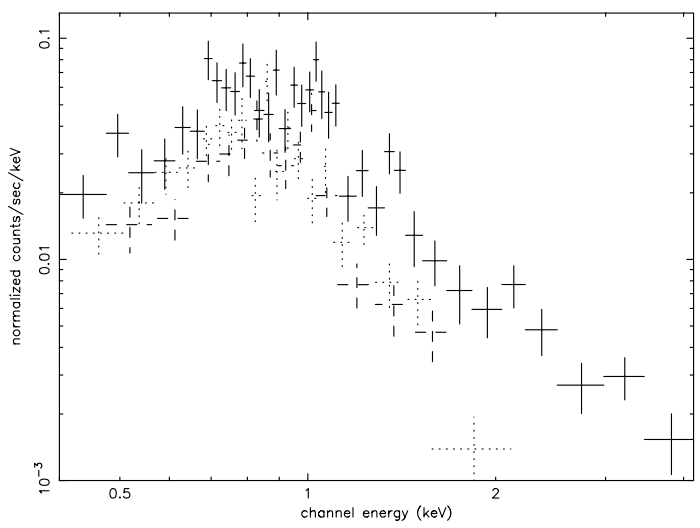

Fig. 7. Spectra of JTH 15510 in the $c$ (dotted lines), $d$ (dashed lines) and $f$ (solid lines) Obs. respectively.

Pleiades (Fig. 6) show very similar amplitudes of variations, and the difference is marginal since the two distributions differ at a $\mathrm{CL} \geq 92 \%$. These results suggest that the amplitude variations both for dF7-dK2 and dK3-dM in NGC 2516 are consistent with the analogous distributions in the coeval Pleiades.

\section{Spectral variability}

Spectral properties of stellar coronae of the cluster have been investigated in Pillitteri et al. (2006) by fitting the X-ray spectra of EPIC/pn simultaneously. They found that spectra of $\mathrm{G}, \mathrm{K}$, and $\mathrm{M}$ type stars are described well with one or two thermal components, similar to other clusters, such as Pleiades (Briggs \& Pye 2003), Blanco 1 (Pillitteri et al. 2004), IC 2391 (Marino et al. 2005), etc.

We searched for spectral variations in the cluster members for which more than one pn spectra were obtainable. Due to low counts for most of the sources, this search was possible only for three stars (Table 5). Two of them are early-type stars for which stellar structure models predict the lack of, or a very thin, convective zone (required to generate magnetic activity), and hence the lack of one key ingredient for an $\alpha-\Omega$ dynamo. Even though no X-ray emission is expected from these stars, the early-type JTH 15509 and JTH 15510 are the brightest sources in the EPIC field of view. JTH 15510, an A0 star flagged as single by Jeffries et al. (1997) in three of the six observations, have sufficient counts (more than 350) to obtain three separate spectra (Fig. 7). 

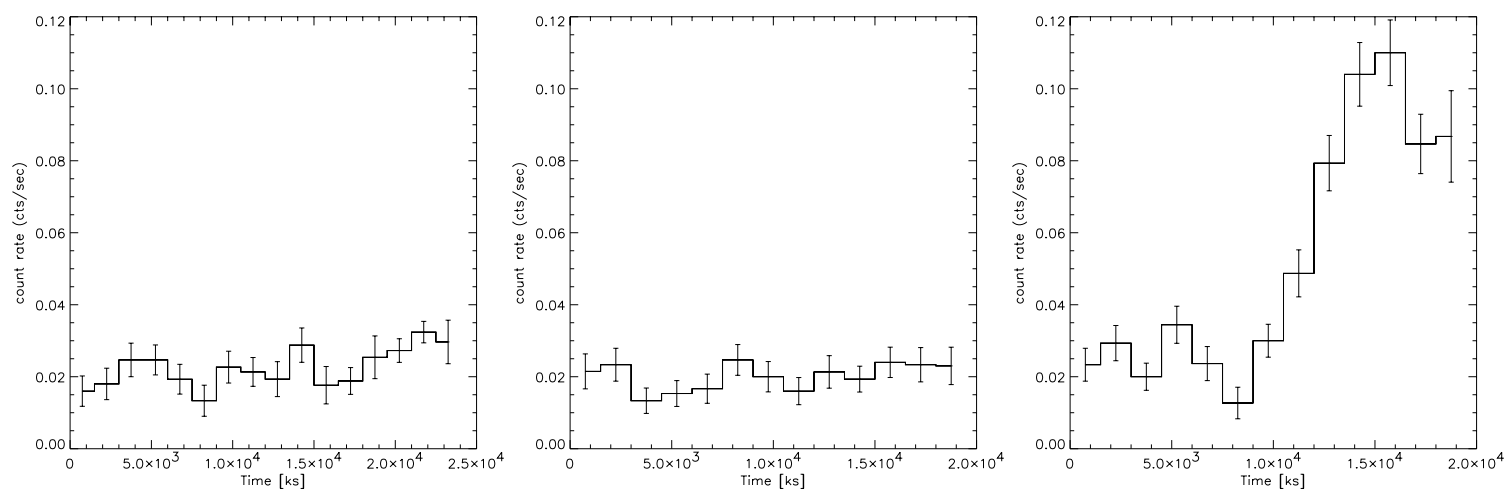

Fig. 8. From the left: light curves of JTH 15510 in the $c, d$ and $f$ Obs. respectively.

Table 5. Stars for which spectral variability search was possible.

\begin{tabular}{ccccr}
\hline \hline JTH & $\begin{array}{c}\text { RA } \\
(\mathrm{J} 2000)\end{array}$ & $\begin{array}{c}\text { Dec } \\
(\mathrm{J} 2000)\end{array}$ & $V$ & $B-V$ \\
\hline 826 & $07: 58: 43.39$ & $-60: 55: 26.8$ & 13.84 & 0.818 \\
15510 & $07: 58: 50.35$ & $-60: 38: 38.9$ & 9.51 & 0.080 \\
15509 & $07: 58: 50.62$ & $-60: 49: 29.6$ & 5.80 & -0.090 \\
\hline
\end{tabular}

The two spectra concerning Obs. $c$ and $d$ are very similar, and simultaneous fits are possible, while the spectrum in the Obs. $f$ is different, implying different physical conditions. As shown in Fig. 8 the spectral variability in Obs. $f$ corresponds to the presence of a flare in the light-curve, while the remaining two lightcurves show no evidence of significant variability. The fits with the 2- $T$ APEC model plus a photometric absorption provides for Obs. $c$ and $d$, similar temperatures at $\approx 0.39 \mathrm{keV}$ and $1.00 \mathrm{keV}$, with $E M_{\text {cold }} / E M_{\text {hot }} \approx 1.2$ having fixed $N_{\mathrm{H}}=6 \times 10^{20} \mathrm{~cm}^{-2}$, and assumed under-solar abundances at $0.3 Z_{\odot}$. To fit Obs. $f$, a third hotter temperature component must be added to the model that fits Obs. $c$ and $d$.

The late type star JTH 826 has sufficient counts to obtain acceptable spectra in two observations (Obs. $a$ and $f$ of Table 1). Part of Obs. $f$ shows a flare that could involve spectral variations. However, this flare interested only a small part $(\approx 10 \%)$ of the observation, and the relatively poor statistic did not allow us to separately fit flaring and quiescent parts of the observation. The spectra in the two observations do not present evidence of spectral variability.

Finally, JTH 15509, a B2 star, is the optically brightest and hottest blue star of the cluster; suitable for a search for spectral variability. Dachs \& Hummel (1996) give for this star an age of the order of one quarter of that estimated for the cluster $\left(\sim 2.5 \times 10^{7} \mathrm{yr}\right)$, suggesting that it is a blue straggler formed by mass transfer from a companion star in a close binary system. The X-ray emission observed in B stars is generally attributed to either intrinsic emission arising from shock heating of the surrounding medium by a high-velocity, radiatively driven wind or to the presence of an active, late-type companion (e.g. Dachs \& Hummel 1996; Cassinelli et al. 1994). Recent results on earlytype stars in the Orion Nebula (Stelzer et al. 2005) hint that X-ray emission mechanism in hot stars is more complex than the simple wind-shock picture, suggesting in particular that magnetic phenomena may be important even in massive stars.

A simultaneous fit of four observations (Obs. $a, c, d, f$ in Table 1) with a sum of two APEC models plus a photometric absorption provides the best fit to the data, as measured with an $\chi^{2}$ test $\left(\chi_{v}^{2}=1.3\right.$ for 82 degrees of freedom). Assuming undersolar abundances at 0.3 and $N_{\mathrm{H}}=6 \times 10^{20} \mathrm{~cm}^{-2}$, we obtained temperatures of $0.91 \mathrm{keV}$ and $3.29 \mathrm{keV}$, with a ratio of the emission measure of $E M_{\text {hot }} / E M_{\text {cold }} \approx 7.49$. No evidence of spectral variability is present.

\section{Summary and conclusions}

We analysed the variability of members of the open cluster NGC 2516 observed with XMM-Newton/EPIC. Using six XMM-Newton/EPIC observations, we explored X-ray variability on short ( $<1$ day), medium (months), and long (years) time scales. We detected 303 distinct detections corresponding to 867 sources, and 474 of these sources were identified with 194 members of the cluster. Stars with spectral type ranging from B-type to M- were most detected.

Variability on short time scales is not very common among NGC 2516 members likely due to the limited statistics of our observations; the Kolmogorov-Smirnov test applied to all X-ray photon time series of detected cluster members shows that only a small fraction $(12 \%)$ of the cluster members are variable at a confidence level greater than $99 \%$, suggesting that the X-ray variability does not depend on stellar mass.

We computed the time distribution function of the X-ray amplitude variations for late-type stars in our sample. This distribution yields the fraction of time that a star spends with a flux higher, by a certain factor, than its minimum value. Our results show that the time XADs on the short and medium time scales of solar-like (dF7-dK2) stars are very alike. A similar result has been found for low-mass stars $(\mathrm{dK} 3-\mathrm{dM})$, suggesting that, on the time scales we explored (from $<1$ day to 17 months), the amplitude of variability does not change with mass. Note that at ages higher than that of this cluster, solar-like stars are less variable than low mass stars (Marino et al. 2002). Comparing our data with the ROSAT/PSPC (on time scales of 7-8 yr) and ROSAT/HRI (on time scales of 4-5 yr) observations of late-type stars of the cluster, we find no evidence for long-term cyclic variability with amplitude similar to the solar one.

The comparison of time XADs for dF7-dK2 and dK3-dM NGC 2516 stars with the corresponding of the coeval Pleiades shows that the amplitude variations for the two samples are consistent. We searched for spectral variability in the cluster members for which more than one spectrum was available. We find evidence of spectral variability only in one star due to a flare in one observation.

Acknowledgements. This work is based on observations obtained by XMM-Newton, an ESA science mission with instruments and contributions directly funded by ESA Member States and the USA (NASA). We acknowledge financial support from MIUR and the anonymous referee. 


\section{References}

Ambruster, C. W., Sciortino, S., \& Golub, L. 1987, ApJS, 65, 273

Baliunas, S. L., Donahue, R. A., Soon, W. H., et al. 1995, ApJ, 438, 269

Barbera, M., Micela, G., Sciortino, S., Harnden, F. R., \& Rosner, R. 1993, ApJ, 414, 846

Briggs, K. R., \& Pye, J. P. 2003, MNRAS, 345, 714

Cassinelli, J. P., Cohen, D. H., Macfarlane, J. J., Sanders, W. T., \& Welsh, B. Y. 1994, ApJ, 421, 705

Dachs, J., \& Hummel, W. 1996, A\&A, 312, 818

Damiani, F., Maggio, A., Micela, G., \& Sciortino, S. 1997a, ApJ, 483, 350

Damiani, F., Maggio, A., Micela, G., \& Sciortino, S. 1997b, ApJ, 483, 370

Damiani, F., Flaccomio, E., Micela, G., et al. 2003, ApJ, 588, 1009

Favata, F., Micela, G., Baliunas, S. L., et al. 2004, A\&A, 418, L13

Franciosini, E., Randich, S., \& Pallavicini, R. 2003, A\&A, 405, 551

Gagné, M., Caillault, J., \& Stauffer, J. R. 1995, ApJ, 450, 217

Harnden, F. R., Adams, N. R., Damiani, F., et al. 2001, ApJ, 547, L141

Hempelmann, A., Schmitt, J. H. M. M., Baliunas, S. L., \& Donahue, R. A. 2003, A\&A, 406, L39

Jeffries, R. D., Thurston, M. R., \& Pye, J. P. 1997, MNRAS, 287, 350

Jeffries, R. D., James, D. J., \& Thurston, M. R. 1998, MNRAS, 300, 550

Jeffries, R. D., Thurston, M. R., \& Hambly, N. C. 2001, A\&A, 375, 863

Kreplin, R. W., Dere, K. P., M., H. D., \& Meekins, J. F. 1977, The solar output and its variations (Colorado University press), 187

Maggio, A., Sciortino, S., Vaiana, G. S., et al. 1987, ApJ, 315, 687

Marino, A., Micela, G., \& Peres, G. 2000, A\&A, 353, 177

Marino, A., Micela, G., Peres, G., \& Sciortino, S. 2002, A\&A, 383, 210

Marino, A., Micela, G., Peres, G., \& Sciortino, S. 2003a, A\&A, 407, L63

Marino, A., Micela, G., Peres, G., \& Sciortino, S. 2003b, A\&A, 406, 629

Marino, A., Micela, G., Peres, G., Pillitteri, I., \& Sciortino, S. 2005, A\&A, 430, 287
Micela, G., \& Marino, A. 2003, A\&A, 404, 637

Micela, G., Sciortino, S., Serio, S., et al. 1985, ApJ, 292, 172

Micela, G., Sciortino, S., Vaiana, G. S., et al. 1990, ApJ, 348, 557

Micela, G., Sciortino, S., Kashyap, V., et al. 1996, ApJS, 102, 75

Micela, G., Sciortino, S., Jeffries, R. D., Thurston, M. R., \& Favata, F. 2000, A\&A, 357, 909

Montmerle, T., Koch-Miramond, L., Falgarone, E., \& Grindlay, J. E. 1983, ApJ, 269,182

Peres, G., Orlando, S., Reale, F., Rosner, R., \& Hudson, H. 2000, ApJ, 528, 537 Pillitteri, I., Micela, G., Sciortino, S., Damiani, F., \& Harnden, F. R. 2004, A\&A, 421,175

Pillitteri, I., Micela, G., Damiani, F., \& Sciortino, S. 2006, A\&A, in press Pillitteri, I., Micela, G., Reale, F., \& Sciortino, S. 2005, A\&A, 430, 155 Pinsonneault, M. H., Stauffer, J., Soderblom, D. R., King, J. R., \& Hanson, R. B. 1998, ApJ, 504, 170

Randich, S., Schmitt, J. H. M. M., Prosser, C. F., \& Stauffer, J. R. 1995, A\&A, 300,134

Read, A. M., \& Ponman, T. J. 2003, A\&A, 409, 395

Robichon, N., Arenou, F., Mermilliod, J.-C., \& Turon, C. 1999, A\&A, 345, 471

Sciortino, S., Damiani, F., Favata, F., Flaccomio, E., \& Micela, G. 2002, in Stellar Coronae in the Chandra and XMM-NEWTON Era, ASP Conf. Ser., 277, 389 Stauffer, J. R., Caillault, J.-P., Gagne, M., Prosser, C. F., \& Hartmann, L. W. 1994, ApJS, 91, 625

Stelzer, B., Flaccomio, E., Montmerle, T., et al. 2005, ApJS, in Press

Stern, R. A., Schmitt, J. H. M. M., \& Kahabka, P. T. 1995, ApJ, 448, 683

Terndrup, D. M., Pinsonneault, M., Jeffries, R. D., et al. 2002, ApJ, 576, 950

Vaiana, G. S., Cassinelli, J. P., Fabbiano, G., et al. 1981, ApJ, 245, 163

Wilson, O. C. 1978, ApJ, 226, 379

Wolk, S. J., Harnden, F. R., Murray, S. S., et al. 2004, ApJ, 606, 466 
A. Marino et al.: X-ray variability of NGC 2516 stars, Online Material p 1

\section{Online Material}


A. Marino et al.: X-ray variability of NGC 2516 stars, Online Material p 2

Table 2. X-ray and optical properties of NGC 2516 members in the XMM-Newton/EPIC/pn observations having more than 25 counts.

\begin{tabular}{|c|c|c|c|c|c|c|c|c|c|}
\hline JTH & Obs. & $\begin{array}{c}\text { RA } \\
\text { J2000 }\end{array}$ & $\begin{array}{c}\text { Dec } \\
\text { J2000 }\end{array}$ & $V$ & $B-V$ & $V-I$ & $\begin{array}{c}\text { Exposure } \\
{[\mathrm{s}]}\end{array}$ & $\begin{array}{c}\text { Rate } \pm \text { err } \\
\text { cnt } / \mathrm{ks}\end{array}$ & $\begin{array}{c}\text { Results of } \\
\text { the K-S test }\end{array}$ \\
\hline 323 & $f$ & $07: 56: 22.80$ & $-60: 51: 42.7$ & 11.556 & 0.513 & 0.659 & 4894.6 & $16.750 \pm 1.850$ & - \\
\hline 15496 & $a$ & 07:56:46.30 & $-60: 48: 59.5$ & 8.360 & 0.020 & & 8094.8 & $4.200 \pm 0.720$ & - \\
\hline 15496 & $b$ & 07:56:46.30 & $-60: 48: 59.5$ & 8.360 & 0.020 & & 5880.3 & $5.100 \pm 0.931$ & - \\
\hline 15496 & $c$ & 07:56:46.30 & $-60: 48: 59.5$ & 8.360 & 0.020 & & 8806.8 & $4.825 \pm 0.740$ & - \\
\hline 15496 & $d$ & $07: 56: 46.30$ & $-60: 48: 59.5$ & 8.360 & 0.020 & & 6764.2 & $5.915 \pm 0.935$ & $90 \%-99 \%$ \\
\hline 15496 & $f$ & 07:56:46.30 & $-60: 48: 59.5$ & 8.360 & 0.020 & & 6030.5 & $5.305 \pm 0.938$ & - \\
\hline 15497 & $a$ & 07:56:46.30 & $-60: 48: 59.5$ & 8.780 & 0.090 & & 8094.8 & $4.200 \pm 0.720$ & - \\
\hline 15497 & $b$ & 07:56:46.30 & $-60: 48: 59.5$ & 8.780 & 0.090 & & 5880.3 & $5.100 \pm 0.931$ & - \\
\hline 15497 & $c$ & $07: 56: 46.30$ & $-60: 48: 59.5$ & 8.780 & 0.090 & & 8806.8 & $4.825 \pm 0.740$ & - \\
\hline 15497 & $d$ & 07:56:46.30 & $-60: 48: 59.5$ & 8.780 & 0.090 & & 6764.2 & $5.915 \pm 0.935$ & $90 \%-99 \%$ \\
\hline 15497 & $f$ & 07:56:46.30 & $-60: 48: 59.5$ & 8.780 & 0.090 & & 6030.5 & $5.305 \pm 0.938$ & - \\
\hline 397 & $c$ & 07:56:47.16 & $-60: 43: 22.1$ & 16.324 & 1.331 & 1.655 & 9381.9 & $7.460 \pm 0.890$ & - \\
\hline 397 & $d$ & 07:56:47.16 & $-60: 43: 22.1$ & 16.324 & 1.331 & 1.655 & 6619.6 & $5.890 \pm 0.940$ & - \\
\hline 397 & $f$ & $07: 56: 47.16$ & $-60: 43: 22.1$ & 16.324 & 1.331 & 1.655 & 6596.5 & $7.280 \pm 1.050$ & $\geq 99 \%$ \\
\hline 400 & $f$ & $07: 56: 47.88$ & $-60: 56: 48.5$ & 10.854 & 0.344 & 0.452 & 4894.6 & $15.940 \pm 1.800$ & - \\
\hline 402 & $a$ & 07:56:48.00 & $-60: 51: 9.5$ & 18.353 & 1.640 & 2.376 & 8729.1 & $3.090 \pm 0.600$ & - \\
\hline 401 & $c$ & $07: 56: 48.26$ & $-60: 46: 33.9$ & 15.410 & 1.268 & 1.545 & 9572.7 & $12.430 \pm 1.140$ & - \\
\hline 401 & $d$ & $07: 56: 48.26$ & $-60: 46: 33.9$ & 15.410 & 1.268 & 1.545 & 7087.5 & $8.180 \pm 1.070$ & - \\
\hline 401 & $a$ & $07: 56: 48.26$ & $-60: 46: 33.9$ & 15.410 & 1.268 & 1.545 & 7313.0 & $7.250 \pm 1.000$ & - \\
\hline 401 & $b$ & $07: 56: 48.26$ & $-60: 46: 33.9$ & 15.410 & 1.268 & 1.545 & 5323.5 & $6.760 \pm 1.130$ & - \\
\hline 401 & $e$ & $07: 56: 48.26$ & $-60: 46: 33.9$ & 15.410 & 1.268 & 1.545 & 2246.7 & $11.130 \pm 2.230$ & - \\
\hline 420 & $c$ & $07: 56: 54.36$ & $-60: 47: 22.1$ & 17.287 & 1.561 & 2.147 & 10091.0 & $3.570 \pm 0.590$ & - \\
\hline 424 & $b$ & 07:56:55.01 & $-60: 58: 11.5$ & 17.316 & 1.511 & 2.006 & 5969.2 & $6.370 \pm 1.030$ & - \\
\hline 432 & $c$ & 07:56:59.62 & $-60: 47: 1.3$ & 9.698 & 0.146 & 0.198 & 10757.0 & $4.740 \pm 0.660$ & $\geq 99 \%$ \\
\hline 432 & $f$ & 07:56:59.62 & $-60: 47: 1.3$ & 9.698 & 0.146 & 0.198 & 7493.5 & $3.870 \pm 0.720$ & - \\
\hline 432 & $a$ & 07:56:59.62 & $-60: 47: 1.3$ & 9.698 & 0.146 & 0.198 & 8514.1 & $4.820 \pm 0.750$ & $\geq 99 \%$ \\
\hline 468 & $c$ & 07:57:10.20 & $-60: 48: 1.5$ & 12.437 & 0.602 & 0.718 & 11899.0 & $5.130 \pm 0.660$ & - \\
\hline 469 & $d$ & 07:57:10.37 & $-60: 44: 10.6$ & 13.330 & & 0.839 & 8868.1 & $1.520 \pm 0.414$ & - \\
\hline 474 & $d$ & 07:57:10.37 & $-60: 44: 10.6$ & 13.289 & 0.732 & 0.843 & 8868.1 & $1.520 \pm 0.414$ & - \\
\hline 483 & $c$ & 07:57:10.92 & $-60: 49: 7$ & 18.397 & 1.499 & 2.371 & 11433.0 & $5.860 \pm 0.720$ & $\geq 99 \%$ \\
\hline 478 & $c$ & 07:57:11.04 & $-60: 39: 36.3$ & 13.852 & 0.891 & 0.995 & 10559.0 & $1.375 \pm 0.361$ & $90 \%-99 \%$ \\
\hline 478 & $d$ & 07:57:11.04 & $-60: 39: 36.3$ & 13.852 & 0.891 & 0.995 & 7272.0 & $1.995 \pm 0.524$ & $90 \%-99 \%$ \\
\hline 481 & $c$ & 07:57:11.04 & $-60: 39: 36.3$ & 13.661 & 0.874 & 0.987 & 10559.0 & $1.375 \pm 0.361$ & $90 \%-99 \%$ \\
\hline 481 & $d$ & 07:57:11.04 & $-60: 39: 36.3$ & 13.661 & 0.874 & 0.987 & 7272.0 & $1.995 \pm 0.524$ & $90 \%-99 \%$ \\
\hline 490 & $d$ & 07:57:12.77 & $-60: 33: 7.3$ & 14.576 & 1.015 & 1.080 & 4950.0 & $15.350 \pm 1.760$ & - \\
\hline 490 & $f$ & 07:57:12.77 & $-60: 33: 7.3$ & 14.576 & 1.015 & 1.080 & 4984.5 & $10.430 \pm 1.450$ & - \\
\hline 492 & $a$ & 07:57:12.89 & $-60: 45: 2.8$ & 20.212 & & 2.968 & 8460.7 & $5.080 \pm 0.780$ & - \\
\hline 496 & $c$ & $07: 57: 14.23$ & $-60: 40: 53$ & 12.240 & 0.674 & 0.769 & 11740.0 & $8.690 \pm 0.860$ & - \\
\hline 496 & $d$ & $07: 57: 14.23$ & $-60: 40: 53$ & 12.240 & 0.674 & 0.769 & 8122.4 & $21.670 \pm 1.630$ & $\geq 99 \%$ \\
\hline 496 & $f$ & 07:57:14.23 & $-60: 40: 53$ & 12.240 & 0.674 & 0.769 & 8409.0 & $12.010 \pm 1.200$ & - \\
\hline 496 & $a$ & 07:57:14.23 & $-60: 40: 53$ & 12.240 & 0.674 & 0.769 & 5799.7 & $9.830 \pm 1.300$ & - \\
\hline 496 & $b$ & $07: 57: 14.23$ & $-60: 40: 53$ & 12.240 & 0.674 & 0.769 & 4391.7 & $12.070 \pm 1.660$ & - \\
\hline 496 & $e$ & $07: 57: 14.23$ & $-60: 40: 53$ & 12.240 & 0.674 & 0.769 & 2550.9 & $14.110 \pm 2.350$ & - \\
\hline 501 & $c$ & 07:57:15.48 & $-60: 38: 55.3$ & 15.035 & 1.068 & 1.200 & 10636.0 & $3.760 \pm 0.590$ & - \\
\hline 501 & $d$ & 07:57:15.48 & $-60: 38: 55.3$ & 15.035 & 1.068 & 1.200 & 7268.2 & $4.400 \pm 0.780$ & - \\
\hline 506 & $c$ & 07:57:16.68 & $-60: 47: 12.7$ & 12.035 & 0.537 & 0.652 & 13034.0 & $4.140 \pm 0.560$ & $90 \%-99 \%$ \\
\hline 15499 & $c$ & 07:57:19.66 & $-60: 48: 43.3$ & 9.290 & 0.120 & & 12773.0 & $5.950 \pm 0.680$ & - \\
\hline 15499 & $d$ & 07:57:19.66 & $-60: 48: 43.3$ & 9.290 & 0.120 & & 10110.0 & $9.300 \pm 0.960$ & - \\
\hline 15499 & $f$ & 07:57:19.66 & $-60: 48: 43.3$ & 9.290 & 0.120 & & 9012.1 & $6.660 \pm 0.860$ & - \\
\hline 15499 & $a$ & 07:57:19.66 & $-60: 48: 43.3$ & 9.290 & 0.120 & & 10944.0 & $7.950 \pm 0.850$ & - \\
\hline 15499 & $b$ & 07:57:19.66 & $-60: 48: 43.3$ & 9.290 & 0.120 & & 8154.9 & $4.170 \pm 0.720$ & - \\
\hline 522 & $c$ & 07:57:20.62 & $-60: 55: 46.4$ & 17.301 & 1.476 & 2.020 & 7939.8 & $5.670 \pm 0.840$ & - \\
\hline
\end{tabular}


Table 2. continued.

\begin{tabular}{|c|c|c|c|c|c|c|c|c|c|}
\hline JTH & Obs. & $\begin{array}{l}\mathrm{RA}_{\text {ott }} \\
\mathrm{J} 2000\end{array}$ & $\begin{array}{l}\text { Dec }_{\text {ott }} \\
\text { J2000 }\end{array}$ & $\bar{V}$ & $B-V$ & $V-I$ & $\begin{array}{c}\text { Exposure } \\
{[\mathrm{s}]}\end{array}$ & $\begin{array}{l}\text { Rate } \pm \text { err } \\
\text { cnt } / \mathrm{ks}\end{array}$ & $\mathrm{K}-\mathrm{S}$ \\
\hline 522 & $e$ & $07: 57: 20.62$ & $-60: 55: 46.4$ & 17.301 & 1.476 & 2.020 & 2240.6 & $11.160 \pm 2.230$ & - \\
\hline 523 & $c$ & 07:57:20.86 & $-60: 44: 0.8$ & 14.945 & 1.065 & 1.204 & 13991.0 & $3.790 \pm 0.520$ & - \\
\hline 523 & $d$ & 07:57:20.86 & $-60: 44: 0.8$ & 14.945 & 1.065 & 1.204 & 10000.0 & $7.200 \pm 0.850$ & - \\
\hline 523 & $f$ & 07:57:20.86 & $-60: 44: 0.8$ & 14.945 & 1.065 & 1.204 & 9982.4 & $6.410 \pm 0.800$ & - \\
\hline 523 & $a$ & 07:57:20.86 & $-60: 44: 0.8$ & 14.945 & 1.065 & 1.204 & 8317.3 & $7.570 \pm 0.950$ & $\geq 99 \%$ \\
\hline 523 & $b$ & 07:57:20.86 & $-60: 44: 0.8$ & 14.945 & 1.065 & 1.204 & 6039.3 & $6.460 \pm 1.030$ & - \\
\hline 530 & $c$ & $07: 57: 23.23$ & $-60: 49: 39.8$ & 11.320 & 0.517 & 0.659 & 12691.0 & $3.780 \pm 0.550$ & - \\
\hline 530 & $d$ & $07: 57: 23.23$ & $-60: 49: 39.8$ & 11.320 & 0.517 & 0.659 & 10283.0 & $6.710 \pm 0.810$ & - \\
\hline 530 & $f$ & $07: 57: 23.23$ & $-60: 49: 39.8$ & 11.320 & 0.517 & 0.659 & 9046.0 & $3.100 \pm 0.580$ & - \\
\hline 530 & $a$ & $07: 57: 23.23$ & $-60: 49: 39.8$ & 11.320 & 0.517 & 0.659 & 12319.0 & $6.010 \pm 0.700$ & - \\
\hline 530 & $b$ & $07: 57: 23.23$ & $-60: 49: 39.8$ & 11.320 & 0.517 & 0.659 & 8979.4 & $4.900 \pm 0.740$ & - \\
\hline 539 & $c$ & $07: 57: 25.13$ & $-60: 46: 51.5$ & 13.047 & 0.684 & 0.794 & 14454.0 & $2.970 \pm 0.450$ & - \\
\hline 540 & $f$ & $07: 57: 25.44$ & $-60: 56: 51.6$ & 15.064 & 1.260 & 1.413 & 5685.5 & $4.920 \pm 0.930$ & - \\
\hline 542 & $c$ & 07:57:26.09 & $-60: 45: 40.4$ & 15.935 & 1.288 & 1.495 & 14839.0 & $4.040 \pm 0.520$ & - \\
\hline 542 & $a$ & 07:57:26.09 & $-60: 45: 40.4$ & 15.935 & 1.288 & 1.495 & 9730.6 & $4.730 \pm 0.700$ & - \\
\hline 542 & $b$ & 07:57:26.09 & $-60: 45: 40.4$ & 15.935 & 1.288 & 1.495 & 7078.9 & $4.240 \pm 0.770$ & - \\
\hline 547 & $c$ & 07:57:28.39 & $-60: 51: 26.6$ & 13.399 & 0.742 & 0.873 & 11805.0 & $6.440 \pm 0.740$ & - \\
\hline 547 & $d$ & 07:57:28.39 & $-60: 51: 26.6$ & 13.399 & 0.742 & 0.873 & 10051.0 & $5.470 \pm 0.740$ & - \\
\hline 547 & $f$ & 07:57:28.39 & $-60: 51: 26.6$ & 13.399 & 0.742 & 0.873 & 8577.4 & $4.430 \pm 0.720$ & - \\
\hline 547 & $a$ & 07:57:28.39 & $-60: 51: 26.6$ & 13.399 & 0.742 & 0.873 & 13686.0 & $2.850 \pm 0.460$ & $90 \%-99 \%$ \\
\hline 547 & $b$ & 07:57:28.39 & $-60: 51: 26.6$ & 13.399 & 0.742 & 0.873 & 9920.6 & $5.440 \pm 0.740$ & - \\
\hline 551 & $f$ & 07:57:29.30 & $-60: 46: 6.6$ & 18.142 & 1.637 & 2.357 & 10542.0 & $3.040 \pm 0.540$ & - \\
\hline 552 & $c$ & 07:57:29.57 & $-60: 50: 12.8$ & 13.643 & 0.812 & 0.960 & 12995.0 & $2.540 \pm 0.440$ & - \\
\hline 552 & $d$ & $07: 57: 29.57$ & $-60: 50: 12.8$ & 13.643 & 0.812 & 0.960 & 10666.0 & $4.310 \pm 0.640$ & - \\
\hline 552 & $f$ & 07:57:29.57 & $-60: 50: 12.8$ & 13.643 & 0.812 & 0.960 & 9385.2 & $20.780 \pm 1.490$ & $\geq 99 \%$ \\
\hline 553 & $d$ & $07: 57: 30.58$ & $-60: 52: 26.0$ & 19.088 & & 2.748 & 9400.1 & $5.430 \pm 0.760$ & $90 \%-99 \%$ \\
\hline 553 & $f$ & 07:57:30.58 & $-60: 52: 26.0$ & 19.088 & & 2.748 & 8331.1 & $19.090 \pm 1.510$ & - \\
\hline 553 & $a$ & $07: 57: 30.58$ & $-60: 52: 26.0$ & 19.088 & & 2.748 & 13444.0 & $4.090 \pm 0.550$ & - \\
\hline 553 & $b$ & $07: 57: 30.58$ & $-60: 52: 26.0$ & 19.088 & & 2.748 & 9549.9 & $3.350 \pm 0.590$ & - \\
\hline 557 & $c$ & 07:57:30.96 & $-60: 38: 27.1$ & 18.058 & 1.661 & 2.183 & 11740.0 & $4.940 \pm 0.650$ & - \\
\hline 556 & $c$ & 07:57:30.98 & $-60: 48: 33.1$ & 14.037 & 0.817 & 0.979 & 14460.0 & $10.930 \pm 0.870$ & - \\
\hline 556 & $d$ & 07:57:30.98 & $-60: 48: 33.1$ & 14.037 & 0.817 & 0.979 & 11513.0 & $13.200 \pm 1.070$ & $90 \%-99 \%$ \\
\hline 556 & $f$ & 07:57:30.98 & $-60: 48: 33.1$ & 14.037 & 0.817 & 0.979 & 10313.0 & $15.220 \pm 1.210$ & - \\
\hline 556 & $a$ & 07:57:30.98 & $-60: 48: 33.1$ & 14.037 & 0.817 & 0.979 & 12444.0 & $5.300 \pm 0.650$ & $90 \%-99 \%$ \\
\hline 556 & $b$ & 07:57:30.98 & $-60: 48: 33.1$ & 14.037 & 0.817 & 0.979 & 8995.5 & $8.120 \pm 0.950$ & - \\
\hline 556 & $e$ & 07:57:30.98 & $-60: 48: 33.1$ & 14.037 & 0.817 & 0.979 & 3658.3 & $9.020 \pm 1.570$ & - \\
\hline 562 & $a$ & 07:57:31.46 & $-60: 59: 31.0$ & 10.899 & 0.329 & & 10338.0 & $14.030 \pm 1.160$ & - \\
\hline 562 & $b$ & 07:57:31.46 & $-60: 59: 31.0$ & 10.899 & 0.329 & & 7540.9 & $15.380 \pm 1.430$ & - \\
\hline 570 & $d$ & 07:57:33.89 & $-60: 50: 43.7$ & 18.731 & 1.600 & 2.436 & 10971.0 & $2.550 \pm 0.480$ & - \\
\hline 576 & $c$ & 07:57:36.29 & $-60: 48: 14.7$ & 13.730 & 0.809 & 0.907 & 15331.0 & $2.020 \pm 0.360$ & - \\
\hline 15500 & $c$ & $07: 57: 37.82$ & $-60: 54: 35.8$ & 8.810 & 0.060 & & 9793.7 & $7.960 \pm 0.900$ & - \\
\hline 15500 & $d$ & $07: 57: 37.82$ & $-60: 54: 35.8$ & 8.810 & 0.060 & & 8852.9 & $8.360 \pm 0.970$ & - \\
\hline 15500 & $f$ & 07:57:37.82 & $-60: 54: 35.8$ & 8.810 & 0.060 & & 7481.0 & $4.010 \pm 0.730$ & - \\
\hline 15500 & $a$ & $07: 57: 37.82$ & $-60: 54: 35.8$ & 8.810 & 0.060 & & 14338.0 & $7.670 \pm 0.730$ & - \\
\hline 15500 & $b$ & $07: 57: 37.82$ & $-60: 54: 35.8$ & 8.810 & 0.060 & & 10429.0 & $7.000 \pm 0.820$ & - \\
\hline 589 & $a$ & 07:57:38.86 & $-61: 02: 8.9$ & 12.759 & 0.649 & 0.778 & 8670.4 & $9.460 \pm 1.040$ & - \\
\hline 589 & $b$ & 07:57:38.86 & $-61: 02: 8.9$ & 12.759 & 0.649 & 0.778 & 6300.2 & $8.730 \pm 1.180$ & - \\
\hline 592 & $d$ & 07:57:39.89 & $-60: 46: 40.1$ & 15.707 & 1.337 & 1.598 & 12865.0 & $2.570 \pm 0.450$ & $\geq 99 \%$ \\
\hline 592 & $f$ & 07:57:39.89 & $-60: 46: 40.1$ & 15.707 & 1.337 & 1.598 & 11959.0 & $2.590 \pm 0.470$ & - \\
\hline 592 & $a$ & 07:57:39.89 & $-60: 46: 40.1$ & 15.707 & 1.337 & 1.598 & 11461.0 & $3.320 \pm 0.540$ & $90 \%-99 \%$ \\
\hline 592 & $e$ & 07:57:39.89 & $-60: 46: 40.1$ & 15.707 & 1.337 & 1.598 & 4091.7 & $6.600 \pm 1.270$ & - \\
\hline 600 & $a$ & 07:57:40.85 & $-60: 52: 33.4$ & 20.218 & & 3.263 & 15058.0 & $3.250 \pm 0.460$ & - \\
\hline 601 & $c$ & 07:57:42.70 & $-60: 44: 21.9$ & 12.682 & 0.636 & 0.750 & 17086.0 & $3.340 \pm 0.440$ & - \\
\hline 601 & $d$ & 07:57:42.70 & $-60: 44: 21.9$ & 12.682 & 0.636 & 0.750 & 12818.0 & $3.280 \pm 0.510$ & - \\
\hline 601 & $f$ & 07:57:42.70 & $-60: 44: 21.9$ & 12.682 & 0.636 & 0.750 & 12784.0 & $2.740 \pm 0.460$ & - \\
\hline 603 & $d$ & 07:57:43.51 & $-60: 45: 7.8$ & 19.565 & & 2.643 & 13078.0 & $2.140 \pm 0.400$ & $90 \%-99 \%$ \\
\hline 615 & $c$ & $07: 57: 47.33$ & $-60: 51: 38.7$ & 16.417 & 1.408 & 1.633 & 13153.0 & $2.810 \pm 0.460$ & - \\
\hline
\end{tabular}


Table 2. continued.

\begin{tabular}{|c|c|c|c|c|c|c|c|c|c|}
\hline JTH & Obs. & $\begin{array}{l}\mathrm{RA}_{\text {ott }} \\
\mathrm{J} 2000\end{array}$ & $\begin{array}{l}\text { Dec }_{\text {ott }} \\
\text { J2000 }\end{array}$ & $\bar{V}$ & $\overline{B-V}$ & $\overline{V-I}$ & $\begin{array}{c}\text { Exposure } \\
{[\mathrm{s}]}\end{array}$ & $\begin{array}{c}\text { Rate } \pm \text { err } \\
\mathrm{cnt} / \mathrm{ks}\end{array}$ & $\overline{\mathrm{K}-\mathrm{S}}$ \\
\hline 15501 & $c$ & $07: 57: 47.69$ & $-60: 36: 35.6$ & 8.390 & -0.010 & & 11316.0 & $6.800 \pm 0.780$ & - \\
\hline 15501 & $f$ & 07:57:47.69 & $-60: 36: 35.6$ & 8.390 & -0.010 & & 8449.1 & $6.860 \pm 0.900$ & - \\
\hline 625 & $c$ & 07:57:51.22 & $-60: 42: 11.9$ & 17.771 & 1.589 & 2.120 & 17025.0 & $2.640 \pm 0.390$ & - \\
\hline 624 & $c$ & $07: 57: 51.50$ & $-60: 43: 17.0$ & 18.702 & & 2.568 & 17728.0 & $6.710 \pm 0.620$ & $90 \%-99 \%$ \\
\hline 624 & $f$ & $07: 57: 51.50$ & $-60: 43: 17.0$ & 18.702 & & 2.568 & 13414.0 & $5.220 \pm 0.620$ & - \\
\hline 635 & $f$ & 07:57:53.38 & $-60: 46: 13.9$ & 19.713 & & 2.866 & 13631.0 & $6.900 \pm 0.710$ & $\geq 99 \%$ \\
\hline 635 & $b$ & $07: 57: 53.38$ & $-60: 46: 13.9$ & 19.713 & & 2.866 & 8245.2 & $3.880 \pm 0.690$ & - \\
\hline 638 & $c$ & 07:57:53.95 & $-60: 45: 6.0$ & 13.806 & 0.837 & 0.921 & 17296.0 & $3.580 \pm 0.460$ & - \\
\hline 638 & $d$ & 07:57:53.95 & $-60: 45: 6.0$ & 13.806 & 0.837 & 0.921 & 14281.0 & $4.200 \pm 0.540$ & - \\
\hline 638 & $a$ & 07:57:53.95 & $-60: 45: 6.0$ & 13.806 & 0.837 & 0.921 & 10633.0 & $2.450 \pm 0.480$ & $90 \%-99 \%$ \\
\hline 643 & $c$ & $07: 57: 55.34$ & $-60: 40: 41.3$ & 12.632 & 0.602 & 0.699 & 16018.0 & $3.000 \pm 0.430$ & - \\
\hline 643 & $d$ & 07:57:55.34 & $-60: 40: 41.3$ & 12.632 & 0.602 & 0.699 & 11282.0 & $4.430 \pm 0.630$ & - \\
\hline 643 & $f$ & $07: 57: 55.34$ & $-60: 40: 41.3$ & 12.632 & 0.602 & 0.699 & 12310.0 & $3.740 \pm 0.550$ & - \\
\hline 643 & $a$ & 07:57:55.34 & $-60: 40: 41.3$ & 12.632 & 0.602 & 0.699 & 7055.0 & $5.390 \pm 0.870$ & $\geq 99 \%$ \\
\hline 642 & $c$ & 07:57:55.44 & $-60: 43: 22.3$ & 13.990 & 0.885 & 0.996 & 18167.0 & $2.370 \pm 0.360$ & - \\
\hline 640 & $c$ & 07:57:55.46 & $-60: 48: 30.0$ & 12.905 & 0.719 & 0.876 & 17024.0 & $12.390 \pm 0.850$ & - \\
\hline 640 & $d$ & $07: 57: 55.46$ & $-60: 48: 30.0$ & 12.905 & 0.719 & 0.876 & 14339.0 & $13.180 \pm 0.960$ & - \\
\hline 640 & $f$ & 07:57:55.46 & $-60: 48: 30.0$ & 12.905 & 0.719 & 0.876 & 13090.0 & $10.700 \pm 0.900$ & $90 \%-99 \%$ \\
\hline 640 & $a$ & 07:57:55.46 & $-60: 48: 30.0$ & 12.905 & 0.719 & 0.876 & 13504.0 & $4.890 \pm 0.600$ & - \\
\hline 640 & $e$ & 07:57:55.46 & $-60: 48: 30.0$ & 12.905 & 0.719 & 0.876 & 4554.0 & $18.880 \pm 2.040$ & - \\
\hline 647 & $f$ & 07:57:56.11 & $-60: 55: 0.2$ & 14.322 & 0.935 & 1.008 & 8104.4 & $3.700 \pm 0.680$ & - \\
\hline 647 & $a$ & 07:57:56.11 & $-60: 55: 0.2$ & 14.322 & 0.935 & 1.008 & 15794.0 & $2.340 \pm 0.390$ & $90 \%-99 \%$ \\
\hline 647 & $b$ & 07:57:56.11 & $-60: 55: 0.2$ & 14.322 & 0.935 & 1.008 & 11487.0 & $3.400 \pm 0.540$ & - \\
\hline 652 & $c$ & 07:57:57.79 & $-60: 44: 15.9$ & 9.893 & 0.195 & 0.251 & 18668.0 & $7.550 \pm 0.640$ & $90 \%-99 \%$ \\
\hline 652 & $d$ & 07:57:57.79 & $-60: 44: 15.9$ & 9.893 & 0.195 & 0.251 & 14167.0 & $6.990 \pm 0.700$ & - \\
\hline 652 & $f$ & 07:57:57.79 & $-60: 44: 15.9$ & 9.893 & 0.195 & 0.251 & 14365.0 & $7.660 \pm 0.730$ & - \\
\hline 652 & $b$ & 07:57:57.79 & $-60: 44: 15.9$ & 9.893 & 0.195 & 0.251 & 7270.6 & $11.000 \pm 1.230$ & - \\
\hline 652 & $e$ & 07:57:57.79 & $-60: 44: 15.9$ & 9.893 & 0.195 & 0.251 & 4485.8 & $8.250 \pm 1.360$ & - \\
\hline 653 & $c$ & 07:57:57.79 & $-60: 53: 40.2$ & 14.429 & 0.928 & 1.052 & 11528.0 & $9.800 \pm 0.920$ & $90 \%-99 \%$ \\
\hline 653 & $d$ & 07:57:57.79 & $-60: 53: 40.2$ & 14.429 & 0.928 & 1.052 & 10778.0 & $8.910 \pm 0.910$ & - \\
\hline 653 & $f$ & 07:57:57.79 & $-60: 53: 40.2$ & 14.429 & 0.928 & 1.052 & 9043.8 & $11.720 \pm 1.140$ & - \\
\hline 653 & $a$ & 07:57:57.79 & $-60: 53: 40.2$ & 14.429 & 0.928 & 1.052 & 16356.0 & $9.170 \pm 0.750$ & - \\
\hline 653 & $b$ & 07:57:57.79 & $-60: 53: 40.2$ & 14.429 & 0.928 & 1.052 & 11976.0 & $7.260 \pm 0.780$ & - \\
\hline 653 & $e$ & 07:57:57.79 & $-60: 53: 40.2$ & 14.429 & 0.928 & 1.052 & 3419.1 & $10.820 \pm 1.780$ & - \\
\hline 655 & $c$ & 07:57:58.39 & $-60: 45: 45.5$ & 15.153 & 1.279 & 1.420 & 18879.0 & $3.810 \pm 0.450$ & - \\
\hline 661 & $c$ & 07:57:59.62 & $-60: 56: 55.9$ & 12.647 & 0.655 & 0.802 & 8460.4 & $17.140 \pm 1.420$ & - \\
\hline 661 & $d$ & 07:57:59.62 & $-60: 56: 55.9$ & 12.647 & 0.655 & 0.802 & 8105.8 & $3.080 \pm 0.620$ & - \\
\hline 661 & $f$ & 07:57:59.62 & $-60: 56: 55.9$ & 12.647 & 0.655 & 0.802 & 6791.9 & $9.280 \pm 1.170$ & - \\
\hline 661 & $a$ & 07:57:59.62 & $-60: 56: 55.9$ & 12.647 & 0.655 & 0.802 & 14429.0 & $10.950 \pm 0.870$ & - \\
\hline 661 & $b$ & 07:57:59.62 & $-60: 56: 55.9$ & 12.647 & 0.655 & 0.802 & 10515.0 & $36.900 \pm 1.870$ & $\geq 99 \%$ \\
\hline 664 & $c$ & 07:58: 0.36 & $-60: 52: 12.0$ & 15.761 & 1.417 & 1.706 & 13249.0 & $16.610 \pm 1.120$ & $\geq 99 \%$ \\
\hline 664 & $d$ & 07:58: 0.36 & $-60: 52: 12.0$ & 15.761 & 1.417 & 1.706 & 12333.0 & $7.460 \pm 0.780$ & $90 \%-99 \%$ \\
\hline 664 & $f$ & 07:58: 0.36 & $-60: 52: 12.0$ & 15.761 & 1.417 & 1.706 & 10544.0 & $5.030 \pm 0.690$ & - \\
\hline 664 & $a$ & 07:58: 0.36 & $-60: 52: 12.0$ & 15.761 & 1.417 & 1.706 & 16123.0 & $5.270 \pm 0.570$ & $90 \%-99 \%$ \\
\hline 664 & $b$ & 07:58: 0.36 & $-60: 52: 12.0$ & 15.761 & 1.417 & 1.706 & 12094.0 & $3.390 \pm 0.530$ & - \\
\hline 671 & $c$ & 07:58: 2.28 & $-60: 46: 47.5$ & 12.068 & 0.603 & 0.741 & 18785.0 & $17.730 \pm 0.970$ & - \\
\hline 671 & $d$ & $07: 58: 2.28$ & $-60: 46: 47.5$ & 12.068 & 0.603 & 0.741 & 15374.0 & $18.280 \pm 1.090$ & $90 \%-99 \%$ \\
\hline 671 & $f$ & $07: 58: 2.28$ & $-60: 46: 47.5$ & 12.068 & 0.603 & 0.741 & 13863.0 & $14.930 \pm 1.040$ & - \\
\hline 671 & $a$ & 07:58: 2.28 & $-60: 46: 47.5$ & 12.068 & 0.603 & 0.741 & 12852.0 & $12.450 \pm 0.980$ & - \\
\hline 671 & $b$ & $07: 58: 2.28$ & $-60: 46: 47.5$ & 12.068 & 0.603 & 0.741 & 9296.3 & $13.660 \pm 1.210$ & - \\
\hline 671 & $e$ & $07: 58: 2.28$ & $-60: 46: 47.5$ & 12.068 & 0.603 & 0.741 & 4886.2 & $19.240 \pm 1.980$ & $90 \%-99 \%$ \\
\hline 15503 & $c$ & $07: 58: 2.66$ & $-60: 48: 48.1$ & 8.950 & 0.080 & & 17219.0 & $5.170 \pm 0.550$ & - \\
\hline 15503 & $d$ & 07:58: 2.66 & $-60: 48: 48.1$ & 8.950 & 0.080 & & 14876.0 & $3.630 \pm 0.490$ & - \\
\hline 15503 & $f$ & 07:58: 2.66 & $-60: 48: 48.1$ & 8.950 & 0.080 & & 13407.0 & $3.360 \pm 0.500$ & - \\
\hline 15503 & $a$ & $07: 58: 2.66$ & $-60: 48: 48.1$ & 8.950 & 0.080 & & 14800.0 & $6.820 \pm 0.680$ & - \\
\hline 15503 & $b$ & 07:58: 2.66 & $-60: 48: 48.1$ & 8.950 & 0.080 & & 10749.0 & $4.370 \pm 0.640$ & - \\
\hline 675 & $d$ & 07:58: 4.46 & $-60: 38: 25.9$ & 18.829 & 1.606 & 2.386 & 9716.3 & $3.090 \pm 0.560$ & - \\
\hline
\end{tabular}


Table 2. continued.

\begin{tabular}{|c|c|c|c|c|c|c|c|c|c|}
\hline JTH & Obs. & $\begin{array}{l}\mathrm{RA}_{\text {ott }} \\
\mathrm{J} 2000\end{array}$ & $\begin{array}{l}\mathrm{Dec}_{\text {ott }} \\
\mathrm{J} 2000\end{array}$ & $V$ & $B-V$ & $V-I$ & $\begin{array}{c}\text { Exposure } \\
{[\mathrm{s}]}\end{array}$ & $\begin{array}{c}\text { Rate } \pm \text { err } \\
\mathrm{cnt} / \mathrm{ks}\end{array}$ & $\mathrm{K}-\mathrm{S}$ \\
\hline 676 & $d$ & 07:58: 4.68 & $-60: 52: 42.7$ & 18.790 & 1.602 & 2.442 & 11801.0 & $5.850 \pm 0.700$ & $\geq 99 \%$ \\
\hline 676 & $f$ & 07:58: 4.68 & $-60: 52: 42.7$ & 18.790 & 1.602 & 2.442 & 10287.0 & $3.890 \pm 0.610$ & - \\
\hline 679 & $c$ & 07:58: 5.40 & $-60: 46: 14.2$ & 14.035 & 1.006 & 1.124 & 19333.0 & $5.070 \pm 0.510$ & $90 \%-99 \%$ \\
\hline 679 & $d$ & 07:58: 5.40 & $-60: 46: 14.2$ & 14.035 & 1.006 & 1.124 & 15578.0 & $3.020 \pm 0.440$ & - \\
\hline 679 & $a$ & 07:58: 5.40 & $-60: 46: 14.2$ & 14.035 & 1.006 & 1.124 & 12181.0 & $15.020 \pm 1.110$ & $\geq 99 \%$ \\
\hline 679 & $b$ & 07:58: 5.40 & $-60: 46: 14.2$ & 14.035 & 1.006 & 1.124 & 8864.7 & $6.660 \pm 0.870$ & - \\
\hline 681 & $d$ & 07:58: 5.59 & $-60: 45: 3.4$ & 12.696 & 0.637 & 0.743 & 15286.0 & $3.140 \pm 0.450$ & - \\
\hline 681 & $f$ & 07:58: 5.59 & $-60: 45: 3.4$ & 12.696 & 0.637 & 0.743 & 14883.0 & $3.230 \pm 0.470$ & - \\
\hline 686 & $c$ & 07:58: 7.03 & $-60: 48: 26.1$ & 17.567 & & 2.412 & 17755.0 & $5.070 \pm 0.530$ & - \\
\hline 686 & $d$ & 07:58: 7.03 & $-60: 48: 26.1$ & 17.567 & & 2.412 & 15405.0 & $4.280 \pm 0.530$ & $90 \%-99 \%$ \\
\hline 686 & $f$ & 07:58: 7.03 & $-60: 48: 26.1$ & 17.567 & & 2.412 & 14058.0 & $4.480 \pm 0.560$ & - \\
\hline 686 & $b$ & 07:58: 7.03 & $-60: 48: 26.1$ & 17.567 & & 2.412 & 10458.0 & $7.650 \pm 0.860$ & $\geq 99 \%$ \\
\hline 686 & $d$ & 07:58: 7.46 & $-60: 48: 25.3$ & 17.567 & & 2.412 & 15405.0 & $4.280 \pm 0.530$ & $90 \%-99 \%$ \\
\hline 686 & $f$ & 07:58: 7.46 & $-60: 48: 25.3$ & 17.567 & & 2.412 & 14058.0 & $4.480 \pm 0.560$ & - \\
\hline 686 & $b$ & 07:58: 7.46 & $-60: 48: 25.3$ & 17.567 & & 2.412 & 10458.0 & $7.650 \pm 0.860$ & $\geq 99 \%$ \\
\hline 691 & $c$ & 07:58: 8.81 & $-60: 44: 38.0$ & 12.220 & 0.560 & 0.676 & 19737.0 & $7.800 \pm 0.630$ & - \\
\hline 691 & $d$ & 07:58: 8.81 & $-60: 44: 38.0$ & 12.220 & 0.560 & 0.676 & 15361.0 & $8.850 \pm 0.760$ & - \\
\hline 691 & $f$ & 07:58: 8.81 & $-60: 44: 38.0$ & 12.220 & 0.560 & 0.676 & 15093.0 & $4.040 \pm 0.520$ & - \\
\hline 691 & $a$ & 07:58: 8.81 & $-60: 44: 38.0$ & 12.220 & 0.560 & 0.676 & 10595.0 & $8.020 \pm 0.870$ & $90 \%-99 \%$ \\
\hline 691 & $b$ & 07:58: 8.81 & $-60: 44: 38.0$ & 12.220 & 0.560 & 0.676 & 7705.2 & $11.160 \pm 1.200$ & - \\
\hline 691 & $e$ & 07:58: 8.81 & $-60: 44: 38.0$ & 12.220 & 0.560 & 0.676 & 4861.0 & $10.290 \pm 1.450$ & - \\
\hline 15504 & $c$ & 07:58: 10.30 & $-60: 51: 58.5$ & 9.510 & 0.210 & & 13858.0 & $9.240 \pm 0.820$ & - \\
\hline 15504 & $d$ & 07:58: 10.30 & $-60: 51: 58.5$ & 9.510 & 0.210 & & 13054.0 & $7.810 \pm 0.770$ & - \\
\hline 15504 & $f$ & 07:58: 10.30 & $-60: 51: 58.5$ & 9.510 & 0.210 & & 11190.0 & $10.460 \pm 0.970$ & - \\
\hline 15504 & $a$ & 07:58: 10.30 & $-60: 51: 58.5$ & 9.510 & 0.210 & & 17296.0 & $9.890 \pm 0.760$ & - \\
\hline 15504 & $b$ & 07:58: 10.30 & $-60: 51: 58.5$ & 9.510 & 0.210 & & 12457.0 & $8.430 \pm 0.820$ & - \\
\hline 15504 & $e$ & 07:58: 10.30 & $-60: 51: 58.5$ & 9.510 & 0.210 & & 4096.8 & $7.320 \pm 1.340$ & - \\
\hline 696 & $c$ & 07:58: 10.85 & $-60: 49: 33.2$ & 12.036 & 0.532 & 0.682 & 16684.0 & $2.040 \pm 0.350$ & - \\
\hline 698 & $d$ & 07:58: 11.83 & $-60: 50: 0.1$ & 13.903 & 0.861 & 0.953 & 14592.0 & $4.590 \pm 0.560$ & - \\
\hline 702 & $c$ & 07:58: 12.26 & $-60: 38: 40.3$ & 11.986 & 0.458 & 0.598 & 14505.0 & $4.690 \pm 0.570$ & - \\
\hline 702 & $d$ & 07:58: 12.26 & $-60: 38: 40.3$ & 11.986 & 0.458 & 0.598 & 10128.0 & $8.590 \pm 0.920$ & $\geq 99 \%$ \\
\hline 702 & $f$ & 07:58: 12.26 & $-60: 38: 40.3$ & 11.986 & 0.458 & 0.598 & 11399.0 & $4.740 \pm 0.640$ & - \\
\hline 712 & $d$ & 07:58: 13.78 & $-60: 45: 20.8$ & 10.117 & 0.149 & 0.168 & 15942.0 & $2.070 \pm 0.360$ & - \\
\hline 15505 & $c$ & 07:58: 14.71 & $-60: 43: 34.0$ & 9.690 & 0.230 & & 19354.0 & $1.525 \pm 0.281$ & - \\
\hline 15505 & $f$ & 07:58: 14.71 & $-60: 43: 34.0$ & 9.690 & 0.230 & & 15489.0 & $2.455 \pm 0.398$ & - \\
\hline 15505 & $b$ & 07:58: 14.71 & $-60: 43: 34.0$ & 9.690 & 0.230 & & 6883.5 & $2.105 \pm 0.553$ & - \\
\hline 711 & $c$ & 07:58: 14.71 & $-60: 43: 34.0$ & 9.710 & 0.214 & 0.285 & 19354.0 & $1.525 \pm 0.281$ & - \\
\hline 711 & $f$ & 07:58: 14.71 & $-60: 43: 34.0$ & 9.710 & 0.214 & 0.285 & 15489.0 & $2.455 \pm 0.398$ & - \\
\hline 711 & $b$ & 07:58: 14.71 & $-60: 43: 34.0$ & 9.710 & 0.214 & 0.285 & 6883.5 & $2.105 \pm 0.553$ & - \\
\hline 714 & $a$ & 07:58: 15.48 & $-60: 50: 28.8$ & 20.802 & & 3.163 & 16263.0 & $2.150 \pm 0.360$ & $\geq 99 \%$ \\
\hline 720 & $a$ & 07:58: 16.56 & $-60: 52: 12.2$ & 18.862 & & 2.514 & 17349.0 & $3.630 \pm 0.460$ & $\geq 99 \%$ \\
\hline 721 & $c$ & 07:58: 16.58 & $-60: 46: 52.4$ & 15.215 & 1.129 & 1.279 & 19249.0 & $2.700 \pm 0.370$ & - \\
\hline 721 & $f$ & 07:58: 16.58 & $-60: 46: 52.4$ & 15.215 & 1.129 & 1.279 & 15599.0 & $2.630 \pm 0.410$ & - \\
\hline 721 & $a$ & 07:58: 16.58 & $-60: 46: 52.4$ & 15.215 & 1.129 & 1.279 & 12921.0 & $2.400 \pm 0.430$ & $90 \%-99 \%$ \\
\hline 725 & $c$ & 07:58: 18.26 & $-60: 45: 14.1$ & 11.465 & 0.533 & 0.665 & 19970.0 & $11.020 \pm 0.740$ & - \\
\hline 725 & $d$ & 07:58: 18.26 & $-60: 45: 14.1$ & 11.465 & 0.533 & 0.665 & 15462.0 & $9.960 \pm 0.800$ & $90 \%-99 \%$ \\
\hline 725 & $f$ & 07:58: 18.26 & $-60: 45: 14.1$ & 11.465 & 0.533 & 0.665 & 16273.0 & $13.700 \pm 0.920$ & - \\
\hline 725 & $a$ & 07:58: 18.26 & $-60: 45: 14.1$ & 11.465 & 0.533 & 0.665 & 11182.0 & $11.720 \pm 1.020$ & - \\
\hline 725 & $b$ & 07:58: 18.26 & $-60: 45: 14.1$ & 11.465 & 0.533 & 0.665 & 8123.9 & $9.110 \pm 1.060$ & - \\
\hline 725 & $e$ & 07:58: 18.26 & $-60: 45: 14.1$ & 11.465 & 0.533 & 0.665 & 5087.8 & $9.240 \pm 1.350$ & - \\
\hline 731 & $c$ & 07:58: 19.15 & $-60: 52: 0.2$ & 15.246 & 1.136 & 1.234 & 13919.0 & $2.800 \pm 0.450$ & $90 \%-99 \%$ \\
\hline 731 & $f$ & 07:58: 19.15 & $-60: 52: 0.2$ & 15.246 & 1.136 & 1.234 & 11553.0 & $2.250 \pm 0.440$ & - \\
\hline 731 & $e$ & 07:58: 19.15 & $-60: 52: 0.2$ & 15.246 & 1.136 & 1.234 & 3848.2 & $8.060 \pm 1.450$ & - \\
\hline 735 & $c$ & 07:58: 19.90 & $-60: 42: 0.2$ & 12.336 & 0.583 & 0.709 & 18046.0 & $11.300 \pm 0.790$ & - \\
\hline 735 & $d$ & 07:58: 19.90 & $-60: 42: 0.2$ & 12.336 & 0.583 & 0.709 & 13716.0 & $8.310 \pm 0.780$ & - \\
\hline 735 & $f$ & 07:58: 19.90 & $-60: 42: 0.2$ & 12.336 & 0.583 & 0.709 & 14664.0 & $9.750 \pm 0.820$ & - \\
\hline 735 & $a$ & 07:58: 19.90 & $-60: 42: 0.2$ & 12.336 & 0.583 & 0.709 & 8163.5 & $6.000 \pm 0.860$ & - \\
\hline
\end{tabular}


Table 2. continued.

\begin{tabular}{|c|c|c|c|c|c|c|c|c|c|}
\hline JTH & Obs. & $\begin{array}{l}\mathrm{RA}_{\text {ott }} \\
\mathrm{J} 2000\end{array}$ & $\begin{array}{l}\text { Dec }_{\text {ott }} \\
\text { J2000 }\end{array}$ & $\bar{V}$ & $B-V$ & $V-I$ & $\begin{array}{c}\text { Exposure } \\
{[\mathrm{s}]}\end{array}$ & $\begin{array}{l}\text { Rate } \pm \text { err } \\
\mathrm{cnt} / \mathrm{ks}\end{array}$ & $\mathrm{K}-\mathrm{S}$ \\
\hline 735 & $b$ & $07: 58: 19.90$ & $-60: 42: 0.2$ & 12.336 & 0.583 & 0.709 & 5925.9 & $10.630 \pm 1.340$ & - \\
\hline 735 & $e$ & 07:58:19.90 & $-60: 42: 0.2$ & 12.336 & 0.583 & 0.709 & 4277.9 & $7.250 \pm 1.300$ & - \\
\hline 737 & $c$ & 07:58:20.16 & $-60: 41: 38.7$ & 12.871 & 0.660 & 0.760 & 17697.0 & $2.030 \pm 0.340$ & - \\
\hline 737 & $d$ & 07:58:20.16 & $-60: 41: 38.7$ & 12.871 & 0.660 & 0.760 & 13716.0 & $8.310 \pm 0.780$ & - \\
\hline 737 & $f$ & 07:58:20.16 & $-60: 41: 38.7$ & 12.871 & 0.660 & 0.760 & 14664.0 & $9.750 \pm 0.820$ & - \\
\hline 737 & $e$ & 07:58:20.16 & $-60: 41: 38.7$ & 12.871 & 0.660 & 0.760 & 4277.9 & $7.250 \pm 1.300$ & - \\
\hline 740 & $a$ & $07: 58: 21.22$ & $-60: 58: 52.0$ & 14.680 & 1.156 & 1.294 & 12726.0 & $2.670 \pm 0.460$ & - \\
\hline 15506 & $a$ & $07: 58: 22.51$ & $-60: 51: 26.0$ & 8.020 & -0.020 & & 15968.0 & $1.595 \pm 0.316$ & $90 \%-99 \%$ \\
\hline 15506 & $b$ & $07: 58: 22.51$ & $-60: 51: 26.0$ & 8.020 & -0.020 & & 11457.0 & $1.615 \pm 0.375$ & - \\
\hline 15506 & $c$ & $07: 58: 22.51$ & $-60: 51: 26.0$ & 8.020 & -0.020 & & 14541.0 & $4.265 \pm 0.542$ & $90 \%-99 \%$ \\
\hline 15506 & $d$ & $07: 58: 22.51$ & $-60: 51: 26.0$ & 8.020 & -0.020 & & 13558.0 & $7.415 \pm 0.740$ & $\geq 99 \%$ \\
\hline 15506 & $e$ & $07: 58: 22.51$ & $-60: 51: 26.0$ & 8.020 & -0.020 & & 4315.4 & $7.185 \pm 1.290$ & - \\
\hline 15506 & $f$ & 07:58:22.51 & $-60: 51: 26.0$ & 8.020 & -0.020 & & 12196.0 & $5.780 \pm 0.688$ & - \\
\hline 15507 & $c$ & $07: 58: 22.51$ & $-60: 51: 26.0$ & 7.180 & 0.010 & & 14541.0 & $4.265 \pm 0.542$ & $90 \%-99 \%$ \\
\hline 15507 & $d$ & $07: 58: 22.51$ & $-60: 51: 26.0$ & 7.180 & 0.010 & & 13558.0 & $7.415 \pm 0.740$ & $\geq 99 \%$ \\
\hline 15507 & $f$ & $07: 58: 22.51$ & $-60: 51: 26.0$ & 7.180 & 0.010 & & 12196.0 & $5.780 \pm 0.688$ & - \\
\hline 15507 & $a$ & $07: 58: 22.51$ & $-60: 51: 26.0$ & 7.180 & 0.010 & & 15968.0 & $1.595 \pm 0.316$ & $90 \%-99 \%$ \\
\hline 15507 & $b$ & $07: 58: 22.51$ & $-60: 51: 26.0$ & 7.180 & 0.010 & & 11457.0 & $1.615 \pm 0.375$ & - \\
\hline 15507 & $e$ & 07:58:22.51 & $-60: 51: 26.0$ & 7.180 & 0.010 & & 4315.4 & $7.185 \pm 1.290$ & - \\
\hline 749 & $a$ & 07:58:22.58 & $-60: 48: 27.2$ & 19.980 & & 3.110 & 14290.0 & $2.940 \pm 0.450$ & - \\
\hline 746 & $c$ & $07: 58: 23.14$ & $-60: 40: 21.9$ & 14.516 & 0.943 & 1.118 & 16270.0 & $16.530 \pm 1.010$ & $\geq 99 \%$ \\
\hline 746 & $d$ & $07: 58: 23.14$ & $-60: 40: 21.9$ & 14.516 & 0.943 & 1.118 & 12487.0 & $18.820 \pm 1.230$ & - \\
\hline 746 & $f$ & $07: 58: 23.14$ & $-60: 40: 21.9$ & 14.516 & 0.943 & 1.118 & 13362.0 & $20.880 \pm 1.250$ & $90 \%-99 \%$ \\
\hline 746 & $a$ & 07:58:23.14 & $-60: 40: 21.9$ & 14.516 & 0.943 & 1.118 & 6868.8 & $13.250 \pm 1.390$ & - \\
\hline 746 & $b$ & 07:58:23.14 & $-60: 40: 21.9$ & 14.516 & 0.943 & 1.118 & 4961.7 & $15.120 \pm 1.750$ & $\geq 99 \%$ \\
\hline 746 & $e$ & $07: 58: 23.14$ & $-60: 40: 21.9$ & 14.516 & 0.943 & 1.118 & 3978.5 & $22.620 \pm 2.380$ & - \\
\hline 747 & $a$ & $07: 58: 23.45$ & $-60: 54: 58.5$ & 14.123 & 1.063 & 1.140 & 16211.0 & $12.555 \pm 0.880$ & $\geq 99 \%$ \\
\hline 747 & $b$ & $07: 58: 23.45$ & $-60: 54: 58.5$ & 14.123 & 1.063 & 1.140 & 11801.0 & $13.090 \pm 1.053$ & $90 \%-99 \%$ \\
\hline 747 & $c$ & 07:58:23.45 & $-60: 54: 58.5$ & 14.123 & 1.063 & 1.140 & 10414.0 & $10.705 \pm 1.014$ & - \\
\hline 747 & $d$ & $07: 58: 23.45$ & $-60: 54: 58.5$ & 14.123 & 1.063 & 1.140 & 10048.0 & $11.495 \pm 1.070$ & - \\
\hline 747 & $e$ & $07: 58: 23.45$ & $-60: 54: 58.5$ & 14.123 & 1.063 & 1.140 & 3207.9 & $12.155 \pm 1.947$ & - \\
\hline 747 & $f$ & 07:58:23.45 & $-60: 54: 58.5$ & 14.123 & 1.063 & 1.140 & 8768.8 & $12.775 \pm 1.207$ & - \\
\hline 748 & $c$ & 07:58:23.45 & $-60: 54: 58.5$ & 14.120 & 1.065 & 1.141 & 10414.0 & $10.705 \pm 1.014$ & - \\
\hline 748 & $d$ & $07: 58: 23.45$ & $-60: 54: 58.5$ & 14.120 & 1.065 & 1.141 & 10048.0 & $11.495 \pm 1.070$ & - \\
\hline 748 & $f$ & $07: 58: 23.45$ & $-60: 54: 58.5$ & 14.120 & 1.065 & 1.141 & 8768.8 & $12.775 \pm 1.207$ & - \\
\hline 748 & $a$ & $07: 58: 23.45$ & $-60: 54: 58.5$ & 14.120 & 1.065 & 1.141 & 16211.0 & $12.555 \pm 0.880$ & $\geq 99 \%$ \\
\hline 748 & $b$ & $07: 58: 23.45$ & $-60: 54: 58.5$ & 14.120 & 1.065 & 1.141 & 11801.0 & $13.090 \pm 1.053$ & $90 \%-99 \%$ \\
\hline 748 & $e$ & $07: 58: 23.45$ & $-60: 54: 58.5$ & 14.120 & 1.065 & 1.141 & 3207.9 & $12.155 \pm 1.947$ & - \\
\hline 751 & $c$ & 07:58:23.81 & $-60: 37: 20.1$ & 12.354 & 0.571 & 0.672 & 12635.0 & $7.440 \pm 0.770$ & - \\
\hline 751 & $d$ & 07:58:23.81 & $-60: 37: 20.1$ & 12.354 & 0.571 & 0.672 & 9292.5 & $12.380 \pm 1.150$ & - \\
\hline 751 & $f$ & 07:58:23.81 & $-60: 37: 20.1$ & 12.354 & 0.571 & 0.672 & 10288.0 & $9.820 \pm 0.980$ & - \\
\hline 767 & $c$ & 07:58:29.95 & $-60: 49: 0.0$ & 16.176 & 1.433 & 1.825 & 16927.0 & $6.850 \pm 0.640$ & $\geq 99 \%$ \\
\hline 767 & $d$ & 07:58:29.95 & $-60: 49: 0.0$ & 16.176 & 1.433 & 1.825 & 15680.0 & $7.910 \pm 0.710$ & - \\
\hline 767 & $f$ & 07:58:29.95 & $-60: 49: 0.0$ & 16.176 & 1.433 & 1.825 & 14601.0 & $6.990 \pm 0.690$ & - \\
\hline 767 & $a$ & 07:58:29.95 & $-60: 49: 0.0$ & 16.176 & 1.433 & 1.825 & 14496.0 & $19.380 \pm 1.160$ & $\geq 99 \%$ \\
\hline 767 & $b$ & 07:58:29.95 & $-60: 49: 0.0$ & 16.176 & 1.433 & 1.825 & 10465.0 & $13.860 \pm 1.150$ & $\geq 99 \%$ \\
\hline 767 & $e$ & 07:58:29.95 & $-60: 49: 0.0$ & 16.176 & 1.433 & 1.825 & 4969.5 & $9.060 \pm 1.350$ & - \\
\hline 766 & $c$ & 07:58:30.10 & $-60: 41: 52.1$ & 9.734 & 0.113 & 0.131 & 15908.0 & $7.170 \pm 0.670$ & - \\
\hline 766 & $d$ & 07:58:30.10 & $-60: 41: 52.1$ & 9.734 & 0.113 & 0.131 & 13821.0 & $14.690 \pm 1.030$ & - \\
\hline 766 & $f$ & 07:58:30.10 & $-60: 41: 52.1$ & 9.734 & 0.113 & 0.131 & 14701.0 & $9.120 \pm 0.790$ & - \\
\hline 766 & $a$ & 07:58:30.10 & $-60: 41: 52.1$ & 9.734 & 0.113 & 0.131 & 7866.9 & $10.680 \pm 1.170$ & $90 \%-99 \%$ \\
\hline 766 & $b$ & $07: 58: 30.10$ & $-60: 41: 52.1$ & 9.734 & 0.113 & 0.131 & 5733.6 & $10.810 \pm 1.370$ & - \\
\hline 766 & $e$ & 07:58:30.10 & $-60: 41: 52.1$ & 9.734 & 0.113 & 0.131 & 4411.1 & $12.920 \pm 1.710$ & - \\
\hline 774 & $c$ & 07:58:31.08 & $-60: 37: 47.7$ & 10.583 & 0.307 & 0.386 & 12896.0 & $5.510 \pm 0.650$ & - \\
\hline 774 & $d$ & 07:58:31.08 & $-60: 37: 47.7$ & 10.583 & 0.307 & 0.386 & 9749.5 & $3.490 \pm 0.600$ & - \\
\hline 774 & $f$ & 07:58:31.08 & $-60: 37: 47.7$ & 10.583 & 0.307 & 0.386 & 10772.0 & $4.180 \pm 0.620$ & $\geq 99 \%$ \\
\hline 776 & $c$ & $07: 58: 31.66$ & $-60: 53: 12.0$ & 17.870 & 1.521 & 2.306 & 12055.0 & $2.985 \pm 0.498$ & - \\
\hline
\end{tabular}


Table 2. continued.

\begin{tabular}{|c|c|c|c|c|c|c|c|c|c|}
\hline JTH & Obs. & $\begin{array}{l}\mathrm{RA}_{\text {ott }} \\
\mathrm{J} 2000\end{array}$ & $\begin{array}{l}\mathrm{Dec}_{\text {ott }} \\
\mathrm{J} 2000\end{array}$ & $V$ & $B-V$ & $V-I$ & $\begin{array}{c}\text { Exposure } \\
{[\mathrm{s}]}\end{array}$ & $\begin{array}{l}\text { Rate } \pm \text { err } \\
\text { cnt } / \text { ks }\end{array}$ & $\mathrm{K}-\mathrm{S}$ \\
\hline 776 & $d$ & $07: 58: 31.66$ & $-60: 53: 12.0$ & 17.870 & 1.521 & 2.306 & 11911.0 & $3.860 \pm 0.569$ & - \\
\hline 776 & $f$ & 07:58:31.66 & $-60: 53: 12.0$ & 17.870 & 1.521 & 2.306 & 10101.0 & $3.020 \pm 0.547$ & - \\
\hline 776 & $a$ & 07:58:31.66 & $-60: 53: 12.0$ & 17.870 & 1.521 & 2.306 & 16396.0 & $4.330 \pm 0.514$ & - \\
\hline 776 & $e$ & 07:58:31.66 & $-60: 53: 12.0$ & 17.870 & 1.521 & 2.306 & 3802.3 & $4.075 \pm 1.035$ & - \\
\hline 780 & $c$ & 07:58:31.66 & $-60: 53: 12.0$ & 14.762 & 0.996 & 1.139 & 12055.0 & $2.985 \pm 0.498$ & - \\
\hline 780 & $d$ & 07:58:31.66 & $-60: 53: 12.0$ & 14.762 & 0.996 & 1.139 & 11911.0 & $3.860 \pm 0.569$ & - \\
\hline 780 & $f$ & 07:58:31.66 & $-60: 53: 12.0$ & 14.762 & 0.996 & 1.139 & 10101.0 & $3.020 \pm 0.547$ & - \\
\hline 780 & $a$ & 07:58:31.66 & $-60: 53: 12.0$ & 14.762 & 0.996 & 1.139 & 16396.0 & $4.330 \pm 0.514$ & - \\
\hline 780 & $e$ & 07:58:31.66 & $-60: 53: 12.0$ & 14.762 & 0.996 & 1.139 & 3802.3 & $4.075 \pm 1.035$ & - \\
\hline 784 & $c$ & 07:58:32.09 & $-60: 53: 53.8$ & 10.508 & 0.226 & 0.250 & 11321.0 & $4.150 \pm 0.610$ & - \\
\hline 784 & $a$ & 07:58:32.09 & $-60: 53: 53.8$ & 10.508 & 0.226 & 0.250 & 16212.0 & $4.130 \pm 0.500$ & $90 \%-99 \%$ \\
\hline 784 & $b$ & 07:58:32.09 & $-60: 53: 53.8$ & 10.508 & 0.226 & 0.250 & 11781.0 & $5.690 \pm 0.690$ & $\geq 99 \%$ \\
\hline 787 & $c$ & 07:58:32.26 & $-60: 59: 52.4$ & 16.758 & 1.454 & 1.757 & 6385.4 & $4.850 \pm 0.870$ & - \\
\hline 785 & $d$ & 07:58:32.33 & $-60: 46: 1.0$ & 14.611 & 0.981 & 1.141 & 16627.0 & $15.880 \pm 0.980$ & - \\
\hline 785 & $f$ & 07:58:32.33 & $-60: 46: 1.0$ & 14.611 & 0.981 & 1.141 & 16625.0 & $10.770 \pm 0.800$ & - \\
\hline 785 & $a$ & 07:58:32.33 & $-60: 46: 1.0$ & 14.611 & 0.981 & 1.141 & 11318.0 & $9.630 \pm 0.920$ & - \\
\hline 785 & $b$ & 07:58:32.33 & $-60: 46: 1.0$ & 14.611 & 0.981 & 1.141 & 8270.5 & $10.040 \pm 1.100$ & - \\
\hline 785 & $e$ & 07:58:32.33 & $-60: 46: 1.0$ & 14.611 & 0.981 & 1.141 & 5299.5 & $25.100 \pm 2.180$ & - \\
\hline 788 & $f$ & 07:58:32.90 & $-60: 48: 52.5$ & 17.528 & 1.565 & 1.974 & 14671.0 & $2.930 \pm 0.450$ & - \\
\hline 789 & $c$ & 07:58:33.07 & $-60: 51: 29.8$ & 18.567 & & 2.630 & 14140.0 & $1.840 \pm 0.360$ & - \\
\hline 789 & $d$ & 07:58:33.07 & $-60: 51: 29.8$ & 18.567 & & 2.630 & 13515.0 & $2.000 \pm 0.380$ & $90 \%-99 \%$ \\
\hline 789 & $a$ & 07:58:33.07 & $-60: 51: 29.8$ & 18.567 & & 2.630 & 15511.0 & $2.390 \pm 0.390$ & $90 \%-99 \%$ \\
\hline 789 & $e$ & 07:58:33.07 & $-60: 51: 29.8$ & 18.567 & & 2.630 & 4279.1 & $8.880 \pm 1.440$ & $\geq 99 \%$ \\
\hline 792 & $c$ & 07:58:33.43 & $-60: 44: 27.9$ & 12.841 & 0.691 & 0.836 & 18563.0 & $15.680 \pm 0.920$ & - \\
\hline 792 & $d$ & 07:58:33.43 & $-60: 44: 27.9$ & 12.841 & 0.691 & 0.836 & 15847.0 & $15.140 \pm 0.980$ & - \\
\hline 792 & $f$ & 07:58:33.43 & $-60: 44: 27.9$ & 12.841 & 0.691 & 0.836 & 16447.0 & $13.680 \pm 0.910$ & - \\
\hline 792 & $a$ & 07:58:33.43 & $-60: 44: 27.9$ & 12.841 & 0.691 & 0.836 & 9981.4 & $16.830 \pm 1.300$ & $90 \%-99 \%$ \\
\hline 792 & $b$ & 07:58:33.43 & $-60: 44: 27.9$ & 12.841 & 0.691 & 0.836 & 7446.8 & $11.950 \pm 1.270$ & - \\
\hline 792 & $e$ & 07:58:33.43 & $-60: 44: 27.9$ & 12.841 & 0.691 & 0.836 & 5052.4 & $9.100 \pm 1.340$ & - \\
\hline 796 & $c$ & 07:58:34.15 & $-60: 38: 23.7$ & 20.279 & & 2.995 & 13415.0 & $2.160 \pm 0.400$ & $\geq 99 \%$ \\
\hline 796 & $f$ & 07:58:34.15 & $-60: 38: 23.7$ & 20.279 & & 2.995 & 11268.0 & $2.660 \pm 0.490$ & - \\
\hline 799 & $a$ & 07:58:34.70 & $-61: 03: 19.6$ & 13.084 & 0.681 & 0.801 & 8182.6 & $5.500 \pm 0.820$ & $90 \%-99 \%$ \\
\hline 799 & $b$ & 07:58:34.70 & $-61: 03: 19.6$ & 13.084 & 0.681 & 0.801 & 5976.0 & $8.030 \pm 1.160$ & - \\
\hline 800 & $b$ & 07:58:35.38 & $-60: 46: 53.9$ & 13.405 & 0.736 & 0.838 & 8872.9 & $11.270 \pm 1.130$ & - \\
\hline 800 & $e$ & 07:58:35.38 & $-60: 46: 53.9$ & 13.405 & 0.736 & 0.838 & 5245.4 & $8.960 \pm 1.310$ & - \\
\hline 800 & $d$ & 07:58:35.83 & $-60: 46: 51.2$ & 13.405 & 0.736 & 0.838 & 16487.0 & $14.860 \pm 0.950$ & - \\
\hline 800 & $f$ & 07:58:35.83 & $-60: 46: 51.2$ & 13.405 & 0.736 & 0.838 & 15021.0 & $8.390 \pm 0.750$ & - \\
\hline 800 & $a$ & 07:58:35.83 & $-60: 46: 51.2$ & 13.405 & 0.736 & 0.838 & 12248.0 & $26.370 \pm 1.470$ & - \\
\hline 800 & $b$ & 07:58:35.83 & $-60: 46: 51.2$ & 13.405 & 0.736 & 0.838 & 8872.9 & $11.270 \pm 1.130$ & - \\
\hline 800 & $e$ & 07:58:35.83 & $-60: 46: 51.2$ & 13.405 & 0.736 & 0.838 & 5245.4 & $8.960 \pm 1.310$ & - \\
\hline 803 & $c$ & 07:58:36.55 & $-60: 50: 17.9$ & 11.675 & 0.585 & 0.695 & 15296.0 & $12.290 \pm 0.900$ & $\geq 99 \%$ \\
\hline 803 & $d$ & 07:58:36.55 & $-60: 50: 17.9$ & 11.675 & 0.585 & 0.695 & 14474.0 & $12.090 \pm 0.910$ & - \\
\hline 803 & $f$ & 07:58:36.55 & $-60: 50: 17.9$ & 11.675 & 0.585 & 0.695 & 12897.0 & $4.960 \pm 0.620$ & - \\
\hline 803 & $a$ & 07:58:36.55 & $-60: 50: 17.9$ & 11.675 & 0.585 & 0.695 & 14915.0 & $6.970 \pm 0.680$ & - \\
\hline 803 & $b$ & 07:58:36.55 & $-60: 50: 17.9$ & 11.675 & 0.585 & 0.695 & 10862.0 & $5.430 \pm 0.710$ & - \\
\hline 803 & $e$ & $07: 58: 36.55$ & $-60: 50: 17.9$ & 11.675 & 0.585 & 0.695 & 4605.1 & $6.730 \pm 1.210$ & - \\
\hline 804 & $f$ & 07:58:36.89 & $-60: 48: 6.8$ & 18.328 & 1.480 & 2.267 & 15229.0 & $3.550 \pm 0.480$ & $90 \%-99 \%$ \\
\hline 806 & $f$ & 07:58:37.10 & $-60: 46: 23.6$ & 15.217 & 1.121 & 1.242 & 16293.0 & $3.680 \pm 0.480$ & - \\
\hline 806 & $b$ & 07:58:37.10 & $-60: 46: 23.6$ & 15.217 & 1.121 & 1.242 & 8482.0 & $7.430 \pm 0.940$ & - \\
\hline 812 & $c$ & 07:58:38.86 & $-60: 48: 23.6$ & 16.885 & 1.441 & 1.837 & 16864.0 & $2.490 \pm 0.380$ & $90 \%-99 \%$ \\
\hline 816 & $c$ & 07:58:40.32 & $-60: 41: 46.0$ & 12.212 & 0.561 & 0.673 & 16350.0 & $2.810 \pm 0.410$ & - \\
\hline 816 & $d$ & 07:58:40.32 & $-60: 41: 46.0$ & 12.212 & 0.561 & 0.673 & 13471.0 & $6.760 \pm 0.710$ & $90 \%-99 \%$ \\
\hline 816 & $f$ & 07:58:40.32 & $-60: 41: 46.0$ & 12.212 & 0.561 & 0.673 & 14456.0 & $4.980 \pm 0.590$ & - \\
\hline 816 & $a$ & 07:58:40.32 & $-60: 41: 46.0$ & 12.212 & 0.561 & 0.673 & 7576.1 & $9.640 \pm 1.130$ & $\geq 99 \%$ \\
\hline 816 & $b$ & 07:58:40.32 & $-60: 41: 46.0$ & 12.212 & 0.561 & 0.673 & 5492.5 & $11.830 \pm 1.470$ & - \\
\hline 820 & $c$ & 07:58:41.78 & $-60: 50: 52.0$ & 17.733 & 1.564 & 2.123 & 14301.0 & $3.220 \pm 0.470$ & - \\
\hline 820 & $d$ & 07:58:41.78 & $-60: 50: 52.0$ & 17.7 & 1.564 & 2.123 & 13841.0 & $3.030 \pm 0.470$ & - \\
\hline
\end{tabular}


Table 2. continued.

\begin{tabular}{|c|c|c|c|c|c|c|c|c|c|}
\hline JTH & Obs. & $\begin{array}{l}\mathrm{RA}_{\text {ott }} \\
\mathrm{J} 2000\end{array}$ & $\begin{array}{l}\text { Dec }_{\text {ott }} \\
\text { J2000 }\end{array}$ & $\bar{V}$ & $B-V$ & $V-I$ & $\begin{array}{c}\text { Exposure } \\
{[\mathrm{s}]}\end{array}$ & $\begin{array}{l}\text { Rate } \pm \text { err } \\
\mathrm{cnt} / \mathrm{ks}\end{array}$ & $\mathrm{K}-\mathrm{S}$ \\
\hline 820 & $f$ & $07: 58: 41.78$ & $-60: 50: 52.0$ & 17.733 & 1.564 & 2.123 & 12899.0 & $4.650 \pm 0.600$ & - \\
\hline 820 & $b$ & 07:58:41.78 & $-60: 50: 52.0$ & 17.733 & 1.564 & 2.123 & 10667.0 & $3.660 \pm 0.590$ & - \\
\hline 823 & $f$ & 07:58:42.84 & $-60: 40: 20.4$ & 13.641 & 0.781 & 0.851 & 13214.0 & $1.970 \pm 0.390$ & - \\
\hline 826 & $c$ & 07:58:43.39 & $-60: 55: 26.8$ & 13.840 & 0.818 & 0.963 & 9528.0 & $16.900 \pm 1.330$ & - \\
\hline 826 & $d$ & 07:58:43.39 & $-60: 55: 26.8$ & 13.840 & 0.818 & 0.963 & 9315.6 & $28.120 \pm 1.740$ & $90 \%-99 \%$ \\
\hline 826 & $f$ & 07:58:43.39 & $-60: 55: 26.8$ & 13.840 & 0.818 & 0.963 & 8385.0 & $66.070 \pm 2.810$ & $\geq 99 \%$ \\
\hline 826 & $a$ & 07:58:43.39 & $-60: 55: 26.8$ & 13.840 & 0.818 & 0.963 & 14433.0 & $40.880 \pm 1.680$ & $90 \%-99 \%$ \\
\hline 826 & $b$ & 07:58:43.39 & $-60: 55: 26.8$ & 13.840 & 0.818 & 0.963 & 10518.0 & $24.530 \pm 1.530$ & - \\
\hline 826 & $e$ & 07:58:43.39 & $-60: 55: 26.8$ & 13.840 & 0.818 & 0.963 & 2969.9 & $28.960 \pm 3.120$ & - \\
\hline 828 & $c$ & 07:58:43.70 & $-60: 32: 57.7$ & 12.607 & 0.762 & 0.864 & 7842.7 & $13.010 \pm 1.290$ & - \\
\hline 828 & $d$ & 07:58:43.70 & $-60: 32: 57.7$ & 12.607 & 0.762 & 0.864 & 5933.9 & $9.610 \pm 1.270$ & - \\
\hline 828 & $e$ & 07:58:43.70 & $-60: 32: 57.7$ & 12.607 & 0.762 & 0.864 & 1890.0 & $15.870 \pm 2.900$ & - \\
\hline 830 & $c$ & 07:58:43.87 & $-60: 45: 36.0$ & 17.859 & 1.597 & 2.263 & 17424.0 & $2.410 \pm 0.370$ & $\geq 99 \%$ \\
\hline 830 & $d$ & 07:58:43.87 & $-60: 45: 36.0$ & 17.859 & 1.597 & 2.263 & 15736.0 & $1.590 \pm 0.320$ & - \\
\hline 840 & $c$ & 07:58:48.22 & $-60: 54: 15.7$ & 14.335 & 0.903 & 1.048 & 10277.0 & $24.330 \pm 1.540$ & - \\
\hline 840 & $d$ & 07:58:48.22 & $-60: 54: 15.7$ & 14.335 & 0.903 & 1.048 & 10331.0 & $17.910 \pm 1.320$ & - \\
\hline 840 & $f$ & 07:58:48.22 & $-60: 54: 15.7$ & 14.335 & 0.903 & 1.048 & 9248.1 & $15.460 \pm 1.290$ & - \\
\hline 840 & $a$ & 07:58:48.22 & $-60: 54: 15.7$ & 14.335 & 0.903 & 1.048 & 14560.0 & $24.930 \pm 1.310$ & $\geq 99 \%$ \\
\hline 840 & $b$ & 07:58:48.22 & $-60: 54: 15.7$ & 14.335 & 0.903 & 1.048 & 10595.0 & $17.460 \pm 1.280$ & $90 \%-99 \%$ \\
\hline 840 & $e$ & 07:58:48.22 & $-60: 54: 15.7$ & 14.335 & 0.903 & 1.048 & 3276.2 & $19.840 \pm 2.460$ & - \\
\hline 845 & $d$ & 07:58:48.84 & $-60: 47: 47.6$ & 18.489 & 1.561 & 2.476 & 15257.0 & $2.880 \pm 0.430$ & - \\
\hline 845 & $a$ & 07:58:48.84 & $-60: 47: 47.6$ & 18.489 & 1.561 & 2.476 & 12104.0 & $4.460 \pm 0.610$ & $90 \%-99 \%$ \\
\hline 843 & $d$ & 07:58:48.89 & $-60: 55: 24.9$ & 11.954 & 0.515 & 0.587 & 9204.5 & $3.040 \pm 0.570$ & - \\
\hline 15510 & $c$ & 07:58:50.35 & $-60: 38: 38.9$ & 9.510 & 0.080 & & 12580.0 & $39.750 \pm 1.780$ & $90 \%-99 \%$ \\
\hline 15510 & $d$ & 07:58:50.35 & $-60: 38: 38.9$ & 9.510 & 0.080 & & 10014.0 & $37.750 \pm 1.940$ & - \\
\hline 15510 & $f$ & 07:58:50.35 & $-60: 38: 38.9$ & 9.510 & 0.080 & & 11126.0 & $85.120 \pm 2.770$ & $\geq 99 \%$ \\
\hline 15510 & $a$ & 07:58:50.35 & $-60: 38: 38.9$ & 9.510 & 0.080 & & 5465.2 & $37.510 \pm 2.620$ & - \\
\hline 15510 & $b$ & 07:58:50.35 & $-60: 38: 38.9$ & 9.510 & 0.080 & & 3971.8 & $36.760 \pm 3.040$ & - \\
\hline 15510 & $e$ & 07:58:50.35 & $-60: 38: 38.9$ & 9.510 & 0.080 & & 3200.7 & $39.370 \pm 3.510$ & - \\
\hline 15509 & $c$ & 07:58:50.62 & $-60: 49: 29.6$ & 5.800 & -0.090 & & 14776.0 & $43.720 \pm 1.720$ & - \\
\hline 15509 & $d$ & 07:58:50.62 & $-60: 49: 29.6$ & 5.800 & -0.090 & & 14265.0 & $31.900 \pm 1.500$ & $\geq 99 \%$ \\
\hline 15509 & $f$ & 07:58:50.62 & $-60: 49: 29.6$ & 5.800 & -0.090 & & 13623.0 & $38.900 \pm 1.690$ & - \\
\hline 15509 & $a$ & 07:58:50.62 & $-60: 49: 29.6$ & 5.800 & -0.090 & & 13202.0 & $49.310 \pm 1.930$ & $\geq 99 \%$ \\
\hline 15509 & $b$ & 07:58:50.62 & $-60: 49: 29.6$ & 5.800 & -0.090 & & 9570.4 & $26.540 \pm 1.670$ & - \\
\hline 15509 & $e$ & 07:58:50.62 & $-60: 49: 29.6$ & 5.800 & -0.090 & & 4530.5 & $39.730 \pm 2.960$ & - \\
\hline 855 & $c$ & 07:58:51.91 & $-60: 35: 20.8$ & 14.781 & 0.988 & 1.127 & 9512.1 & $13.670 \pm 1.200$ & - \\
\hline 855 & $d$ & 07:58:51.91 & $-60: 35: 20.8$ & 14.781 & 0.988 & 1.127 & 7383.2 & $13.140 \pm 1.330$ & - \\
\hline 855 & $f$ & 07:58:51.91 & $-60: 35: 20.8$ & 14.781 & 0.988 & 1.127 & 8193.0 & $14.520 \pm 1.330$ & - \\
\hline 855 & $e$ & 07:58:51.91 & $-60: 35: 20.8$ & 14.781 & 0.988 & 1.127 & 2321.4 & $14.650 \pm 2.510$ & - \\
\hline 856 & $c$ & 07:58:52.51 & $-60: 53: 38.2$ & 12.142 & 0.659 & 0.758 & 10672.0 & $8.250 \pm 0.880$ & - \\
\hline 856 & $d$ & $07: 58: 52.51$ & $-60: 53: 38.2$ & 12.142 & 0.659 & 0.758 & 10672.0 & $7.780 \pm 0.850$ & - \\
\hline 856 & $f$ & 07:58:52.51 & $-60: 53: 38.2$ & 12.142 & 0.659 & 0.758 & 9749.9 & $8.000 \pm 0.910$ & - \\
\hline 856 & $a$ & 07:58:52.51 & $-60: 53: 38.2$ & 12.142 & 0.659 & 0.758 & 14142.0 & $7.280 \pm 0.720$ & $90 \%-99 \%$ \\
\hline 856 & $b$ & 07:58:52.51 & $-60: 53: 38.2$ & 12.142 & 0.659 & 0.758 & 10281.0 & $6.610 \pm 0.800$ & - \\
\hline 856 & $e$ & 07:58:52.51 & $-60: 53: 38.2$ & 12.142 & 0.659 & 0.758 & 3389.3 & $8.260 \pm 1.560$ & - \\
\hline 860 & $c$ & 07:58:52.94 & $-60: 36: 11.8$ & 9.781 & 0.185 & 0.239 & 10115.0 & $11.270 \pm 1.060$ & - \\
\hline 860 & $d$ & 07:58:52.94 & $-60: 36: 11.8$ & 9.781 & 0.185 & 0.239 & 8000.8 & $12.120 \pm 1.230$ & - \\
\hline 860 & $f$ & 07:58:52.94 & $-60: 36: 11.8$ & 9.781 & 0.185 & 0.239 & 8825.6 & $14.390 \pm 1.280$ & - \\
\hline 860 & $e$ & 07:58:52.94 & $-60: 36: 11.8$ & 9.781 & 0.185 & 0.239 & 2550.5 & $11.760 \pm 2.150$ & - \\
\hline 864 & $a$ & 07:58:53.38 & $-60: 56: 33.8$ & 18.389 & 1.562 & 2.624 & 12862.0 & $2.640 \pm 0.450$ & $90 \%-99 \%$ \\
\hline 864 & $b$ & 07:58:53.38 & $-60: 56: 33.8$ & 18.389 & 1.562 & 2.624 & 9289.2 & $4.950 \pm 0.730$ & - \\
\hline 862 & $c$ & 07:58:53.93 & $-60: 54: 23.7$ & 16.856 & 1.527 & 2.088 & 9953.0 & $3.620 \pm 0.600$ & - \\
\hline 862 & $d$ & 07:58:53.93 & $-60: 54: 23.7$ & 16.856 & 1.527 & 2.088 & 9906.4 & $7.970 \pm 0.900$ & $90 \%-99 \%$ \\
\hline 862 & $f$ & 07:58:53.93 & $-60: 54: 23.7$ & 16.856 & 1.527 & 2.088 & 8954.2 & $5.700 \pm 0.800$ & $90 \%-99 \%$ \\
\hline 862 & $a$ & 07:58:53.93 & $-60: 54: 23.7$ & 16.856 & 1.527 & 2.088 & 13868.0 & $5.190 \pm 0.610$ & $90 \%-99 \%$ \\
\hline 862 & $b$ & 07:58:53.93 & $-60: 54: 23.7$ & 16.856 & 1.527 & 2.088 & 10117.0 & $5.040 \pm 0.710$ & - \\
\hline 868 & $d$ & $07: 58: 56.50$ & $-60: 47: 22.3$ & 17.183 & 1.492 & 1.930 & 14544.0 & $1.790 \pm 0.350$ & $90 \%-99 \%$ \\
\hline
\end{tabular}


Table 2. continued.

\begin{tabular}{|c|c|c|c|c|c|c|c|c|c|}
\hline JTH & Obs. & $\begin{array}{l}\mathrm{RA}_{\text {ott }} \\
\mathrm{J} 2000\end{array}$ & $\begin{array}{l}\text { Dec }_{\text {ott }} \\
\text { J2000 }\end{array}$ & $V$ & $B-V$ & $V-I$ & $\begin{array}{c}\text { Exposure } \\
{[\mathrm{s}]}\end{array}$ & $\begin{array}{c}\text { Rate } \pm \text { err } \\
\text { cnt } / \mathrm{ks}\end{array}$ & $\mathrm{K}-\mathrm{S}$ \\
\hline 868 & $b$ & $07: 58: 56.50$ & $-60: 47: 22.3$ & 17.183 & 1.492 & 1.930 & 8064.1 & $4.840 \pm 0.770$ & - \\
\hline 870 & $c$ & 07:58:57.17 & $-60: 36: 13.6$ & 14.867 & 1.034 & 1.171 & 9930.8 & $10.880 \pm 1.050$ & - \\
\hline 870 & $d$ & 07:58:57.17 & $-60: 36: 13.6$ & 14.867 & 1.034 & 1.171 & 7865.9 & $6.610 \pm 0.920$ & - \\
\hline 870 & $f$ & 07:58:57.17 & $-60: 36: 13.6$ & 14.867 & 1.034 & 1.171 & 8677.2 & $10.030 \pm 1.070$ & - \\
\hline 871 & $a$ & 07:58:57.74 & $-60: 43: 3.6$ & 14.760 & 0.990 & 1.140 & 7847.0 & $26.700 \pm 1.845$ & $\geq 99 \%$ \\
\hline 871 & $c$ & 07:58:57.74 & $-60: 43: 3.6$ & 14.760 & 0.990 & 1.140 & 15135.0 & $9.975 \pm 0.812$ & $90 \%-99 \%$ \\
\hline 871 & $b$ & 07:58:57.74 & $-60: 43: 3.6$ & 14.760 & 0.990 & 1.140 & 5703.3 & $7.275 \pm 1.129$ & - \\
\hline 871 & $d$ & 07:58:57.74 & $-60: 43: 3.6$ & 14.760 & 0.990 & 1.140 & 13353.0 & $8.165 \pm 0.782$ & $\geq 99 \%$ \\
\hline 871 & $e$ & 07:58:57.74 & $-60: 43: 3.6$ & 14.760 & 0.990 & 1.140 & 4225.0 & $6.980 \pm 1.285$ & - \\
\hline 871 & $f$ & 07:58:57.74 & $-60: 43: 3.6$ & 14.760 & 0.990 & 1.140 & 14204.0 & $7.005 \pm 0.702$ & - \\
\hline 873 & $c$ & 07:58:57.74 & $-60: 43: 3.6$ & 19.089 & & 2.804 & 15135.0 & $9.975 \pm 0.812$ & $90 \%-99 \%$ \\
\hline 873 & $d$ & 07:58:57.74 & $-60: 43: 3.6$ & 19.089 & & 2.804 & 13353.0 & $8.165 \pm 0.782$ & $\geq 99 \%$ \\
\hline 873 & $f$ & 07:58:57.74 & $-60: 43: 3.6$ & 19.089 & & 2.804 & 14204.0 & $7.005 \pm 0.702$ & - \\
\hline 873 & $a$ & 07:58:57.74 & $-60: 43: 3.6$ & 19.089 & & 2.804 & 7847.0 & $26.700 \pm 1.845$ & $\geq 99 \%$ \\
\hline 873 & $b$ & 07:58:57.74 & $-60: 43: 3.6$ & 19.089 & & 2.804 & 5703.3 & $7.275 \pm 1.129$ & - \\
\hline 873 & $e$ & 07:58:57.74 & $-60: 43: 3.6$ & 19.089 & & 2.804 & 4225.0 & $6.980 \pm 1.285$ & - \\
\hline 884 & $f$ & 07:59:02.78 & $-60: 47: 46.9$ & 10.781 & 0.281 & 0.328 & 13896.0 & $2.450 \pm 0.420$ & $\geq 99 \%$ \\
\hline 896 & $c$ & 07:59:06.02 & $-60: 46: 17.4$ & 19.981 & & 3.002 & 14197.0 & $1.900 \pm 0.370$ & - \\
\hline 896 & $d$ & 07:59:06.02 & $-60: 46: 17.4$ & 19.981 & & 3.002 & 13538.0 & $1.920 \pm 0.380$ & - \\
\hline 897 & $d$ & 07:59: 7.10 & $-60: 52: 1.1$ & 15.874 & 1.255 & 1.467 & 11002.0 & $4.000 \pm 0.600$ & - \\
\hline 907 & $d$ & $07: 59: 10.25$ & $-60: 50: 53.1$ & 17.516 & 1.442 & 2.082 & 11589.0 & $2.420 \pm 0.460$ & - \\
\hline 907 & $f$ & 07:59:10.25 & $-60: 50: 53.1$ & 17.516 & 1.442 & 2.082 & 11094.0 & $4.240 \pm 0.620$ & - \\
\hline 928 & $d$ & 07:59:15.98 & $-60: 47: 38.0$ & 16.266 & 1.495 & 1.850 & 12312.0 & $2.270 \pm 0.430$ & - \\
\hline 933 & $c$ & 07:59:18.00 & $-60: 45: 21.2$ & 17.771 & 1.625 & 2.378 & 12475.0 & $3.050 \pm 0.490$ & - \\
\hline 933 & $d$ & 07:59:18.00 & $-60: 45: 21.2$ & 17.771 & 1.625 & 2.378 & 12017.0 & $4.080 \pm 0.580$ & - \\
\hline 933 & $f$ & 07:59:18.00 & $-60: 45: 21.2$ & 17.771 & 1.625 & 2.378 & 12667.0 & $2.450 \pm 0.440$ & - \\
\hline 933 & $a$ & 07:59:18.00 & $-60: 45: 21.2$ & 17.771 & 1.625 & 2.378 & 8088.9 & $3.090 \pm 0.620$ & - \\
\hline 940 & $c$ & 07:59:19.70 & $-60: 34: 43.4$ & 11.936 & 0.525 & 0.653 & 7437.6 & $58.490 \pm 2.800$ & $\geq 99 \%$ \\
\hline 940 & $d$ & 07:59:19.70 & $-60: 34: 43.4$ & 11.936 & 0.525 & 0.653 & 6053.5 & $5.620 \pm 0.960$ & - \\
\hline 940 & $f$ & 07:59:19.70 & $-60: 34: 43.4$ & 11.936 & 0.525 & 0.653 & 6843.2 & $6.140 \pm 0.950$ & - \\
\hline 944 & $d$ & $07: 59: 21.26$ & $-60: 50: 53.4$ & 19.283 & & 2.881 & 10422.0 & $10.750 \pm 1.020$ & $\geq 99 \%$ \\
\hline 944 & $a$ & $07: 59: 21.26$ & $-60: 50: 53.4$ & 19.283 & & 2.881 & 10414.0 & $3.170 \pm 0.550$ & - \\
\hline 944 & $b$ & $07: 59: 21.26$ & $-60: 50: 53.4$ & 19.283 & & 2.881 & 7520.5 & $5.450 \pm 0.850$ & $\geq 99 \%$ \\
\hline 945 & $c$ & 07:59:21.31 & $-60: 46: 3.2$ & 12.341 & 0.569 & 0.695 & 12057.0 & $4.480 \pm 0.610$ & - \\
\hline 945 & $d$ & 07:59:21.31 & $-60: 46: 3.2$ & 12.341 & 0.569 & 0.695 & 11714.0 & $5.720 \pm 0.700$ & - \\
\hline 945 & $f$ & 07:59:21.31 & $-60: 46: 3.2$ & 12.341 & 0.569 & 0.695 & 11693.0 & $3.930 \pm 0.580$ & - \\
\hline 945 & $a$ & $07: 59: 21.31$ & $-60: 46: 3.2$ & 12.341 & 0.569 & 0.695 & 8143.4 & $12.400 \pm 1.230$ & $\geq 99 \%$ \\
\hline 945 & $b$ & 07:59:21.31 & $-60: 46: 3.2$ & 12.341 & 0.569 & 0.695 & 5923.3 & $5.400 \pm 0.960$ & - \\
\hline 15512 & $c$ & 07:59:21.46 & $-60: 48: 57.9$ & 9.110 & 0.030 & & 11042.0 & $19.560 \pm 1.330$ & $90 \%-99 \%$ \\
\hline 15512 & $d$ & 07:59:21.46 & $-60: 48: 57.9$ & 9.110 & 0.030 & & 10704.0 & $14.850 \pm 1.180$ & $90 \%-99 \%$ \\
\hline 15512 & $f$ & 07:59:21.46 & $-60: 48: 57.9$ & 9.110 & 0.030 & & 11358.0 & $17.960 \pm 1.260$ & $\geq 99 \%$ \\
\hline 15512 & $a$ & 07:59:21.46 & $-60: 48: 57.9$ & 9.110 & 0.030 & & 9480.3 & $19.090 \pm 1.420$ & - \\
\hline 15512 & $b$ & 07:59:21.46 & $-60: 48: 57.9$ & 9.110 & 0.030 & & 6909.1 & $28.220 \pm 2.020$ & - \\
\hline 15512 & $e$ & 07:59:21.46 & $-60: 48: 57.9$ & 9.110 & 0.030 & & 3475.9 & $10.930 \pm 1.770$ & - \\
\hline 947 & $c$ & 07:59:21.67 & $-60: 46: 47.7$ & 14.509 & 0.976 & 1.061 & 11781.0 & $3.060 \pm 0.510$ & - \\
\hline 947 & $d$ & 07:59:21.67 & $-60: 46: 47.7$ & 14.509 & 0.976 & 1.061 & 11465.0 & $2.180 \pm 0.440$ & - \\
\hline 950 & $c$ & 07:59:22.39 & $-60: 45: 27.1$ & 11.873 & 0.616 & & 11841.0 & $8.190 \pm 0.830$ & - \\
\hline 950 & $d$ & 07:59:22.39 & $-60: 45: 27.1$ & 11.873 & 0.616 & & 11525.0 & $7.900 \pm 0.830$ & - \\
\hline 950 & $f$ & 07:59:22.39 & $-60: 45: 27.1$ & 11.873 & 0.616 & & 11521.0 & $2.690 \pm 0.480$ & $\geq 99 \%$ \\
\hline 950 & $a$ & 07:59:22.39 & $-60: 45: 27.1$ & 11.873 & 0.616 & & 7810.1 & $5.510 \pm 0.840$ & - \\
\hline 954 & $c$ & 07:59:24.05 & $-60: 34: 34.1$ & 17.699 & 1.610 & 2.308 & 7049.8 & $4.110 \pm 0.760$ & - \\
\hline 963 & $c$ & 07:59:27.19 & $-60: 47: 55.0$ & 9.639 & 0.075 & 0.084 & 10273.0 & $3.990 \pm 0.620$ & $\geq 99 \%$ \\
\hline 963 & $d$ & 07:59:27.19 & $-60: 47: 55.0$ & 9.639 & 0.075 & 0.084 & 10724.0 & $6.060 \pm 0.750$ & - \\
\hline 963 & $f$ & 07:59:27.19 & $-60: 47: 55.0$ & 9.639 & 0.075 & 0.084 & 10914.0 & $4.860 \pm 0.670$ & - \\
\hline 963 & $a$ & 07:59:27.19 & $-60: 47: 55.0$ & 9.639 & 0.075 & 0.084 & 8471.4 & $5.430 \pm 0.800$ & $\geq 99 \%$ \\
\hline 963 & $b$ & 07:59:27.19 & $-60: 47: 55.0$ & 9.639 & 0.075 & 0.084 & 6166.3 & $9.730 \pm 1.260$ & - \\
\hline 963 & $e$ & 07:59:27.19 & $-60: 47: 55.0$ & 9.639 & 0.075 & 0.084 & 3425.1 & $8.470 \pm 1.570$ & $90 \%-99 \%$ \\
\hline
\end{tabular}


A. Marino et al.: X-ray variability of NGC 2516 stars, Online Material p 10

Table 2. continued.

\begin{tabular}{clcccccccc}
\hline \hline JTH & Obs. & $\begin{array}{c}\text { RA }_{\text {ott }} \\
\text { J2000 }\end{array}$ & $\begin{array}{c}\text { Dec } \\
\text { Jtt }\end{array}$ & $V$ & $B-V$ & $V-I$ & $\begin{array}{c}\text { Exposure } \\
{[\mathrm{s}]}\end{array}$ & $\begin{array}{c}\text { Rate } \pm \text { err } \\
\text { cnt/ks }\end{array}$ & K-S \\
\hline 967 & $c$ & $07: 59: 28.97$ & $-60: 39: 36.8$ & 17.410 & 1.520 & 2.006 & 9534.9 & $2.940 \pm 0.550$ & $\geq 99 \%$ \\
977 & $c$ & $07: 59: 32.04$ & $-60: 48: 47.2$ & 13.879 & 0.834 & 1.019 & 9887.9 & $27.510 \pm 1.670$ & $90 \%-99 \%$ \\
977 & $d$ & $07: 59: 32.04$ & $-60: 48: 47.2$ & 13.879 & 0.834 & 1.019 & 9696.1 & $22.380 \pm 1.520$ & $90 \%-99 \%$ \\
977 & $f$ & $07: 59: 32.04$ & $-60: 48: 47.2$ & 13.879 & 0.834 & 1.019 & 10348.0 & $20.680 \pm 1.410$ & - \\
977 & $a$ & $07: 59: 32.04$ & $-60: 48: 47.2$ & 13.879 & 0.834 & 1.019 & 8466.0 & $28.470 \pm 1.830$ & - \\
977 & $b$ & $07: 59: 32.04$ & $-60: 48: 47.2$ & 13.879 & 0.834 & 1.019 & 6154.6 & $27.620 \pm 2.120$ & - \\
977 & $e$ & $07: 59: 32.04$ & $-60: 48: 47.2$ & 13.879 & 0.834 & 1.019 & 3150.8 & $18.410 \pm 2.420$ & $90 \%-99 \%$ \\
979 & $a$ & $07: 59: 32.04$ & $-60: 58: 48.9$ & 14.995 & 1.048 & 1.177 & 7890.8 & $5.830 \pm 0.860$ & - \\
979 & $b$ & $07: 59: 32.04$ & $-60: 58: 48.9$ & 14.995 & 1.048 & 1.177 & 5727.6 & $7.330 \pm 1.130$ & - \\
999 & $c$ & $07: 59: 40.03$ & $-60: 42: 33.8$ & 14.774 & 1.046 & 1.206 & 9379.7 & $6.180 \pm 0.810$ & $90 \%-99 \%$ \\
999 & $d$ & $07: 59: 40.03$ & $-60: 42: 33.8$ & 14.774 & 1.046 & 1.206 & 8672.4 & $5.190 \pm 0.770$ & - \\
999 & $f$ & $07: 59: 40.03$ & $-60: 42: 33.8$ & 14.774 & 1.046 & 1.206 & 9363.2 & $8.440 \pm 0.950$ & - \\
1012 & $f$ & $07: 59: 47.52$ & $-60: 44: 52.7$ & 17.354 & 1.489 & 2.040 & 8957.6 & $5.360 \pm 0.770$ & - \\
1012 & $f$ & $07: 59: 48.43$ & $-60: 44: 53.7$ & 17.354 & 1.489 & 2.040 & 8957.6 & $5.360 \pm 0.770$ & - \\
1035 & $c$ & $07: 59: 55.78$ & $-60: 41: 53.7$ & 10.780 & 0.318 & 0.395 & 7594.7 & $8.430 \pm 1.050$ & $\geq 99 \%$ \\
1035 & $d$ & $07: 59: 55.78$ & $-60: 41: 53.7$ & 10.780 & 0.318 & 0.395 & 7138.8 & $5.320 \pm 0.860$ & - \\
1035 & $f$ & $07: 59: 55.78$ & $-60: 41: 53.7$ & 10.780 & 0.318 & 0.395 & 7750.9 & $4.640 \pm 0.770$ & - \\
1035 & $a$ & $07: 59: 55.78$ & $-60: 41: 53.7$ & 10.780 & 0.318 & 0.395 & 5105.8 & $10.770 \pm 1.450$ & $\geq 99 \%$ \\
1040 & $c$ & $07: 59: 56.90$ & $-60: 49: 27.8$ & 11.648 & 0.623 & 0.615 & 7234.6 & $4.700 \pm 0.810$ & - \\
1040 & $a$ & $07: 59: 56.90$ & $-60: 49: 27.8$ & 11.648 & 0.623 & 0.615 & 6421.4 & $4.050 \pm 0.790$ & - \\
1040 & $b$ & $07: 59: 56.90$ & $-60: 49: 27.8$ & 11.648 & 0.623 & 0.615 & 4677.5 & $5.990 \pm 1.130$ & - \\
1054 & $a$ & $08: 00: 2.45$ & $-60: 53: 51.1$ & 19.568 & & 2.953 & 6416.5 & $9.820 \pm 1.240$ & $\geq 99 \%$ \\
1097 & $c$ & $08: 00: 20.57$ & $-60: 42: 35.7$ & 13.488 & 0.927 & 1.051 & 5936.9 & $9.770 \pm 1.280$ & - \\
\hline
\end{tabular}

